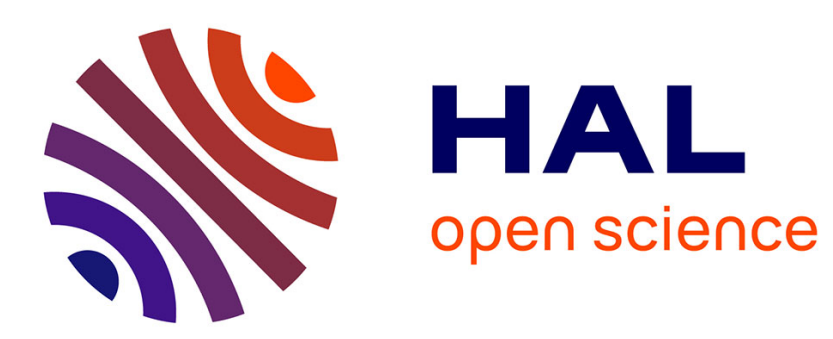

\title{
Solutions to inverse moment estimation problems in dimension 2, using best constrained approximation
}

\author{
Juliette Leblond, Elodie Pozzi
}

\section{To cite this version:}

Juliette Leblond, Elodie Pozzi. Solutions to inverse moment estimation problems in dimension 2, using best constrained approximation. Journal of Approximation Theory, In press, 10.1016/j.jat.2020.105520 . hal-02066780

\section{HAL Id: hal-02066780 \\ https://hal.inria.fr/hal-02066780}

Submitted on 26 Mar 2019

HAL is a multi-disciplinary open access archive for the deposit and dissemination of scientific research documents, whether they are published or not. The documents may come from teaching and research institutions in France or abroad, or from public or private research centers.
L'archive ouverte pluridisciplinaire HAL, est destinée au dépôt et à la diffusion de documents scientifiques de niveau recherche, publiés ou non, émanant des établissements d'enseignement et de recherche français ou étrangers, des laboratoires publics ou privés. 


\title{
Solutions to inverse moment estimation problems in dimension 2, using best constrained approximation
}

\author{
Juliette Leblond* $\quad$ Elodie Pozzi ${ }^{\dagger}$
}

\begin{abstract}
We study an inverse problem that consists in estimating the first (zero-order) moment of some $\mathbb{R}^{2}$-valued distribution $\mathbf{m}$ supported within a closed interval $\bar{S} \subset \mathbb{R}$, from partial knowledge of the solution to the Poisson-Laplace partial differential equation with source term equal to the divergence of $\mathbf{m}$ on another interval parallel to and located at some distance from $S$. Such a question coincides with a $2 \mathrm{D}$ version of an inverse magnetic "net" moment recovery question that arises in paleomagnetism, for thin rock samples. We formulate and constructively solve a best approximation problem under constraint in $L^{2}$ and in Sobolev spaces involving the restriction of the Poisson extension of the divergence of $\mathbf{m}$. Numerical results obtained from the described algorithms for the net moment approximation are also furnished.
\end{abstract}

Keywords: Poisson-Laplace partial differential equation, inverse problems, regularization, best constrained approximation and bounded extremal problems, Poisson and Hilbert transforms, Hardy spaces, Maxwell's equations and issues.

\section{Introduction}

The present study concerns the practical issue of estimating the "net" moment (or the mean value) of some $\mathbb{R}^{2}$-valued distribution (or integrable, or square-integrable function) supported on an interval of the real line, from the partial knowledge of the divergence of its Poisson extension to the upper half-plane, on some interval located on a parallel line at distance $h>0$ from the first one.

This is a two dimensional (2D) formulation of a three dimensional (3D) inverse magnetic moment recovery problem in paleomagnetism, for thin rock samples. There, the magnetization is assumed to be some $\mathbb{R}^{3}$-valued distribution (or integrable function) supported on a planar sample (square), of which we aim at estimating the net moment, given measurements of the normal component of the generated magnetic field on another planar measurements set taken to be a square parallel to the sample and located at some distance $h$ to it. Moment and more general magnetization recovery issues are considered in $[5,6,7]$. More about the data acquisition process and the use of scanning SQUID (Superconducting Quantum Interference Device) microscopy devices for measuring a component of the magnetic field produced by weakly magnetized pieces of rocks can be found in the introductory sections of these references.

In both situations, the partial differential equation (PDE) model that drives the behaviour of the magnetic field derives from Maxwell equations in magnetostatics, see [15]. They ensure that the magnetic field $\mathbf{B}$ derives from a scalar magnetic potential $U$ which is solution to

\footnotetext{
${ }^{*}$ INRIA, Team Factas, 2004 route des Lucioles, 06902 Sophia Antipolis, France

${ }^{\dagger}$ Saint Louis University, Department of Mathematics and Statistics, Ritter Hall, 1N. Grand Blvd., Saint Louis, MO 63103, USA
} 
a Poisson-Laplace elliptic PDE with right-hand side of divergence form, that relates the Laplacian of that potential to the divergence of the magnetization distribution $\mathbf{m}$ :

$$
\mathbf{B}=-\mu_{0} \operatorname{grad} U, \Delta U=\operatorname{div} \mathbf{m} \text { in } \mathbb{R}^{n},
$$

with $n=2,3$, where both $\mathbf{B}, \mathbf{m}$ are $\mathbb{R}^{n}$-valued quantities, and $\mu_{0}$ is the permeability of the free space. We stick to situations where $\mathbf{m}$ has a compact support contained in the hyperplane $\left\{x_{n}=0\right\}$ and measurements of the vertical component $b_{n}$ of the field $\mathbf{B}$ are available on another compact subset of $\left\{x_{n}=h\right\}$ for some $h>0$.

The determination of the magnetic moment (mean value of the magnetization) provides useful preliminary information for the full inversion, i.e. for magnetization estimation, in particular in unidirectional situations where its components are proportional one to the others (the unknown quantity being a $\mathbb{R}$-valued distribution, the direction/orientation vector being fixed).

In 3D situation, these issues were efficiently analyzed with tools from harmonic analysis, specifically Poisson kernel and Riesz transforms [27], in [5, 6, 7], see also references therein, using their links with Hardy spaces of harmonic gradients. The existence of silent sources in $3 \mathrm{D}$, elements of the non-zero kernel of the non injective magnetization-to-field operator (the operator that maps the magnetization to the measured component of the magnetic field) was established together with their characterization in [5]. This makes non unique the solution to the inverse magnetization issue from the corresponding field data. The mean value of the magnetization however is uniquely determined by the field data.

In the present 2D case, we will use similar tools, Poisson kernel, Hilbert transform and harmonic conjugation, links with Hardy spaces of functions of the complex variable [13, 14, 23]; see also [21, 25] for related issues. Considering square-summable magnetizations m supported on an interval $S$ of the real line, we will establish that the magnetization-to-field operator $\mathbf{m} \mapsto b_{2}[\mathbf{m}]$ is injective, whence there are no silent sources, and that the mean value $\langle\mathbf{m}\rangle$ of the magnetization is yet uniquely determined by the field data $b_{2}[\mathbf{m}]$, provided by values of the vertical component on the measurement interval $K$.

Our purpose is to establish the existence and to build linear estimators for the mean values $\left\langle m_{i}\right\rangle$ on $S$ of the components $m_{i}$ of $\mathbf{m}$ for $i=1,2$, as was done in [6] for the 3D case. Indeed, though an academic version of the related physical issue, the present $2 \mathrm{D}$ situation possesses its own mathematical interest and specificity. These linear estimators consist in square summable functions $\phi_{i}$ such that their effect (scalar product) against the data on $K$ is as close as possible to the moment components $\left\langle m_{i}\right\rangle$, for all $\mathbf{m}$ bounded by some $L^{2}$ norm (the functions being built once and for all). With $\mathbf{e}_{1}=\left(\chi_{S}, 0\right), \mathbf{e}_{2}=\left(0, \chi_{S}\right)$, observe that: $\left\langle m_{i}\right\rangle=\left\langle\mathbf{m}, \mathbf{e}_{i}\right\rangle_{L^{2}\left(S, \mathbb{R}^{2}\right)}$, whence, if $b_{2}^{*}$ denotes the adjoint operator to $b_{2}$, we have:

$$
\left|\left\langle b_{2}[\mathbf{m}], \phi_{i}\right\rangle_{L^{2}(K)}-\left\langle m_{i}\right\rangle\right| \leq\|\mathbf{m}\|_{L^{2}\left(S, \mathbb{R}^{2}\right)}\left\|b_{2}^{*}\left[\phi_{i}\right]-\mathbf{e}_{i}\right\|_{L^{2}\left(S, \mathbb{R}^{2}\right)},
$$

where $\phi_{i}$ is a square integrable function on $K$. We will see that the above minimization problem is still ill-posed, even if uniqueness is granted. Specifically, there exist a sequence of functions such that their scalar product on $K$ against $b_{2}[\mathbf{m}]$ converges to $\left\langle m_{i}\right\rangle$ as $n \rightarrow \infty$. Their quadratic norm however diverge, which reflects an unstable behaviour, as is classical in such inverse problems, see [1]. Regularization is thus needed (of Tykhonov type), see [20] in order to set up and to solve a well-posed problem.

In order to construct such a numerical magnetometer, we then face the best constrained approximation issue (bounded extremal problem, BEP) of finding the function $\phi_{i}$ on $K$ satisfying some norm constraint there, such that its scalar product against $b_{2}[\mathbf{m}]$ is as close as possible to $\left\langle m_{i}\right\rangle$.

Such a norm constraint will be considered both in the Lebesgue space $L^{2}(K)$ and in the Sobolev space $W_{0}^{1,2}(K) \subset W^{1,2}(K)$. In those Hilbert spaces, the above problems will be shown to be well-posed, the approximation subsets being closed and convex. The results obtained in $L^{2}(K)$ and in $W_{0}^{1,2}(K)$ are different. In particular, the solutions in $W_{0}^{1,2}(K)$ demonstrate less oscillations at the endpoints of $K$ than the ones in $L^{2}(K)$, and allow 
to incorporate vanishing boundary conditions. The results for moment estimation will be compared between them.

Preliminary numerical computations in the 3D setting, with planar squared support and measurement set, were run on appropriate finite elements bases, see [6]; they are actually heavy. In the present 2D setting and on intervals, they are of course much less costly and we perform some of them using expansions on the Fourier bases, in Lebesgue and Sobolev spaces.

The overview of the present work is as follows. In Section 2, we introduce some notation, recall definitions and properties, concerning Lebesgue, Sobolev and Hardy spaces of functions, together with integral Poisson and Hilbert transforms. We then study in Section 3 the properties of the operator $b_{2}: \mathbf{m} \rightarrow b_{2}[\mathbf{m}]$, which maps the magnetization to the second (vertical) component of the produced magnetic field, and of its adjoint. Section 4 is devoted to the bounded extremal problems of which we consider two versions, in $L^{2}(K)$ and in $W_{0}^{1,2}(K)$, establishing their well-posedness and characterizing their solutions. Computational algorithms together with results of preliminary numerical simulations are provided in Section 5, with figures in an appendix. We finally discuss in Section 6 some concluding remarks and perspectives.

\section{Preliminaries}

Let $\mathbb{C}_{+} \simeq \mathbb{R}_{+}^{2}$ be the upper half-plane $\mathbb{C}_{+}=\{x+i y: y>0\} \subset \mathbb{C}, \mathbb{R}_{+}^{2}=\{(x, y): y>0\} \subset$ $\mathbb{R}^{2}$. The partial derivatives with respect to $x$ and $y$ will be denoted by $\partial_{x}$ and $\partial_{y}$ respectively.

\subsection{Lebesgue and Sobolev spaces}

Let $I \subseteq \mathbb{R}$ a non empty open interval. For $1 \leq p<\infty$, we will denote by $L^{p}(I, \mathbb{K})$ with $\mathbb{K}=\mathbb{R}, \mathbb{C}$ or $\mathbb{R}^{2}$ the space of $\mathbb{K}$-valued functions $f$ such that $|f|^{p}$ is integrable on $I$; we will simply write $L^{p}(I)=L^{p}(I, \mathbb{R})$.

We equip the space $L^{2}\left(I, \mathbb{R}^{2}\right)$ with the following inner product: for $f=\left(f_{1}, f_{2}\right)$ and $g=$ $\left(g_{1}, g_{2}\right) \in L^{2}\left(I, \mathbb{R}^{2}\right)$,

$$
\langle f, g\rangle_{L^{2}\left(I, \mathbb{R}^{2}\right)}=\left\langle\left(f_{1}, f_{2}\right),\left(g_{1}, g_{2}\right)\right\rangle_{L^{2}\left(I, \mathbb{R}^{2}\right)}=\left\langle f_{1}, g_{1}\right\rangle_{L^{2}(I)}+\left\langle f_{2}, g_{2}\right\rangle_{L^{2}(I)},
$$

while $L^{2}(I, \mathbb{C})$ is equipped with:

$$
\langle f, g\rangle_{L^{2}(I, \mathbb{C})}=\langle\operatorname{Re} f, \operatorname{Re} g\rangle_{L^{2}(I)}+\langle\operatorname{Im} f, \operatorname{Im} g\rangle_{L^{2}(I)} \text { for } f, g \in L^{2}(I, \mathbb{C}) .
$$

For $I \subsetneq \mathbb{R}$, for $\phi \in L^{2}(I)$, we will write $\widetilde{\phi}=\phi \vee 0 \in L^{2}(\mathbb{R})$, the function equal to $\phi$ on $I$ and equal to 0 on $\mathbb{R} \backslash I$. Note that we identify $\widehat{L^{2}(I)}$ to $\chi_{I} L^{2}(\mathbb{R})$ and to the subspace of $L^{2}(\mathbb{R})$ of functions supported on $I$. We also have $\widetilde{\phi}_{\left.\right|_{I}}=R_{I} \widetilde{\phi}=\phi$ for $\phi \in L^{2}(I)$ and the adjoint of the restriction operator $R_{I}$ from $L^{2}(\mathbb{R})$ to $L^{2}(I)$ is the extension operator ? on $L^{2}(I)$.

When $I$ is bounded, $\widetilde{L^{2}(I)} \subset L^{2}(\mathbb{R}) \cap L^{1}(\mathbb{R})$. Moreover, functions in $\widetilde{L^{2}(I)}$ are compactly supported on $\mathbb{R}$.

For $I \subseteq \mathbb{R}$, the Sobolev space $W^{1,2}(I)$ is the space of real-valued functions $f \in L^{2}(I)$ such that their (distributional) derivative $f^{\prime}$ belongs to $L^{2}(I) ; W^{1,2}(I)$ is equipped with the norm (see [9, Sec. 8.2]):

$$
\|f\|_{W^{1,2}(I)}=\|f\|_{L^{2}(I)}+\left\|f^{\prime}\right\|_{L^{2}(I)}, \quad f \in W^{1,2}(I) .
$$

A function in $W^{1,2}(I)$ can be extended to a function in $W^{1,2}(\mathbb{R})$, see the extension operator in $[9$, Thm 8.6]. The space $C(\bar{I})$ denotes the space of continuous functions on $I$ with the norm

$$
\|f\|_{C(\bar{I})}=\sup _{x \in \bar{I}}|f(x)|, \quad f \in C(\bar{I}) .
$$

Note that $W^{1,2}(I) \subset C(\bar{I})$, the space of continuous real-valued functions on $\bar{I}$, with compact (hence continuous) injection, see $\left[9\right.$, Thm 8.5]. If $I$ is bounded, $C^{1}(\bar{I}) \subset W^{1,2}(I)$ will denote 
the space of functions in $C(\bar{I})$ with (strong) derivative in $C(\bar{I})$. The space is equipped with the norm

$$
\|f\|_{C^{1}(\bar{I})}=\sup _{x \in \bar{I}}|f(x)|+\sup _{x \in \bar{I}}\left|f^{\prime}(x)\right|, \quad f \in C^{1}(\bar{I}),
$$

where the (strong) derivative coincides with the distributional derivative. The space $C^{\infty}(\mathbb{R})$ will denote the space of functions such that derivatives at all order are continuous. The subspace $W_{0}^{1,2}(I)$ of $W^{1,2}(I)$ is the collection of functions $f \in W^{1,2}(I)$ such that $f=0$ at the endpoints of $I$. A consequence is that $\widetilde{f} \in W^{1,2}(\mathbb{R})$ for $f \in W_{0}^{1,2}(I)$; in other words, . is the extension operator from $W_{0}^{1,2}(I)$ to $W^{1,2}(\mathbb{R})$ (see [9, Sec 8.3, Remark 16]. We equip $W_{0}^{1,2}(K)$ with the norm:

$$
\|\phi\|_{W_{0}^{1,2}(K)}=\left\|\phi^{\prime}\right\|_{L^{2}(K)}, \quad \phi \in W_{0}^{1,2}(K),
$$

which is equivalent to $\|\phi\|_{W^{1,2}(K)}$ by Poincaré's inequality (see [9, Prop 8.13]).

\subsection{Poisson kernel, conjugate Poisson kernel and Hilbert transform}

For $y>0$, we denote by $P_{y}$ the Poisson kernel of the upper half-plane and by $Q_{y}$ the conjugate Poisson kernel. For $x \in \mathbb{R}$ :

$$
P_{y}(x)=\frac{1}{\pi} \frac{y}{x^{2}+y^{2}}, \quad Q_{y}(x)=\frac{1}{\pi} \frac{x}{x^{2}+y^{2}} .
$$

see $[13,14,27]$. Let $x \in \mathbb{R}, y>0$. Then

$$
P_{y} \star u(x)=\frac{1}{\pi} \int_{-\infty}^{\infty} \frac{y}{(x-t)^{2}+y^{2}} u(t) d t, u \in L^{2}(\mathbb{R}),
$$

and $\mathcal{P}_{y}: u \mapsto P_{y} \star u$ is a bounded (real linear) operator from $L^{2}(\mathbb{R})$ to $L^{2}(\mathbb{R})([27$, Thm $1.3])$.

The map, $x \mapsto P_{y}(x)$ belongs to $L^{1}(\mathbb{R})$ (where its norm is equal to 1 ). Moreover, $(x, y) \mapsto$ $P_{y}(x)$ belongs to $C^{\infty}\left(\mathbb{R}_{+}^{2}\right)$. Therefore, its partial derivatives are also $C^{\infty}\left(\mathbb{R}_{+}^{2}\right)$-smooth functions, whence for $y>0, x \mapsto\left(\partial_{x} P_{y}\right)(x)$ and $x \mapsto\left(\partial_{y} P_{y}\right)(x)$ belong to $C^{\infty}(\mathbb{R})$. The functions $P_{y}$ and $Q_{y}$ satisfy the Cauchy-Riemann equations on $\mathbb{C}_{+}: \partial_{y} P_{y}=-\partial_{x} Q_{y}, \partial_{x} P_{y}=\partial_{y} Q_{y}$.

It can be checked by direct computations that $x \mapsto\left(\partial_{x} P_{y}\right)(x)$ and $x \mapsto\left(\partial_{y} P_{y}\right)(x)$ belong to $L^{1}(\mathbb{R})$, and $\left\|\partial_{x} P_{y}\right\|_{L^{1}(\mathbb{R})}=\left\|\partial_{y} P_{y}\right\|_{L^{1}(\mathbb{R})}=2 /(\pi y)$. Hence, $u \rightarrow\left(\partial_{x} P_{y}\right) \star u$ and $u \rightarrow\left(\partial_{y} P_{y}\right) \star u$ are bounded operators from $L^{2}(\mathbb{R})$ to $L^{2}(\mathbb{R})$. Further, for $u \in L^{2}(\mathbb{R})$, $(x, y) \mapsto \mathcal{P}_{y}[u](x)=\left(P_{y} \star u\right)(x)$ is also harmonic in $\mathbb{R}_{+}^{2}$, hence a $C^{\infty}\left(\mathbb{R}_{+}^{2}\right)$-smooth function. Thus, its partial derivatives $x \mapsto \partial_{x}\left(P_{y} \star u\right)(x)$ and $x \mapsto \partial_{y}\left(P_{y} \star u\right)(x)$ belong to $C^{\infty}(\mathbb{R})$, for $y>0$.

Using the Fourier transform as in [7, Sec. 2.2], we see that convolution by the Poisson kernel and differentiation commute: for $y>0$ and $u \in L^{2}(\mathbb{R})$, we have that $\partial_{x}\left(P_{y} \star u\right)=\left(\partial_{x} P_{y}\right) \star u$. In particular, the operator $\mathcal{P}_{y}$ maps continuously $L^{2}(\mathbb{R})$ onto $W^{1,2}(\mathbb{R})$. By Cauchy-Riemann equations, $Q_{y}$ satisfies the properties of $P_{y}$ described above. Indeed, $u \mapsto \partial_{x}\left(Q_{y} \star u\right)$ and $u \mapsto \partial_{y}\left(Q_{y} \star u\right)$ are bounded on $L^{2}(\mathbb{R})$ and for $y>0, x \mapsto \partial_{x}\left(Q_{y} \star u\right)$ and $x \mapsto \partial_{y}\left(Q_{y} \star u\right)$ are $C^{\infty}(\mathbb{R})$.

In order to simplify the notations, we may not use any parentheses in expressions like $\partial_{x} P_{y} \star u, \partial_{y} P_{y} \star u, \partial_{x} Q_{y} \star u$ and $\partial_{y} Q_{y} \star u$ for $u \in L^{2}(\mathbb{R})$ and $y>0$.

Let $I, J \subsetneq \mathbb{R}$ be non empty open bounded interval. Then, for $y>0, R_{I} \mathcal{P}_{y}$ is a HilbertSchmidt operator on $\widetilde{L^{2}(J, \mathbb{C})} \subset L^{2}(\mathbb{R}, \mathbb{C})$ (see [18, p. 264, Ex. 2.19]). Indeed, for $u \in L^{2}(J)$, we have

$$
R_{I} \mathcal{P}_{y}[u](x)=R_{I}\left(P_{y} \star u\right)(x)=\frac{1}{\pi} \int_{J} \frac{h}{(x-t)^{2}+y^{2}} u(t) d t, x \in I,
$$


and $R_{I} \mathcal{P}_{y}$ is an integral operator on $\widetilde{L^{2}(J, \mathbb{C})}$ with kernel $k(x, t)=P_{y}(x-t), k \in L^{2}(I \times J)$, since $P_{y}(x)$ is a smooth function for $y>0$. Thus, it is compact. The same conclusion holds for the operator $u \mapsto R_{I}\left(\partial_{x} P_{y} \star u\right)$ (and $\left.u \mapsto R_{I}\left(\partial_{y} P_{y} \star u\right)\right)$ on $\widetilde{L^{2}(J, \mathbb{C})}$.

For $u \in L^{2}(\mathbb{R})$, the functions $\mathcal{P}_{y}[u]=P_{y} \star u$ and $Q_{y} \star u$ have non-tangential limits as $y$ tends to zero and

$$
\lim _{y \rightarrow 0} P_{y} \star u(x)=u(x) \text { and } \lim _{y \rightarrow 0} Q_{y} \star u(x)=\mathcal{H} u(x),
$$

where $\mathcal{H}$ is the Hilbert transform from $L^{2}(\mathbb{R})$ to $L^{2}(\mathbb{R})$ defined by

$$
\mathcal{H} u(x)=\frac{1}{\pi} \lim _{\varepsilon \rightarrow 0} \int_{|x-t|>\varepsilon} \frac{u(t)}{x-t} d t .
$$

The Hilbert transform is bounded and isometric on $L^{2}(\mathbb{R})$ and satisfies $\mathcal{H}^{2}=-I$, whence its adjoint $\mathcal{H}^{*}=-\mathcal{H}$. The Hilbert transform commutes with the Poisson operator (which can be proved using the Fourier transform):

$$
\mathcal{H}\left(P_{y} \star u\right)=\mathcal{H}\left(\mathcal{P}_{y}[u]\right)=P_{y} \star \mathcal{H} u=\mathcal{P}_{y}[\mathcal{H} u]
$$

For $y>0, x \in \mathbb{R}$ and $u \in L^{2}(\mathbb{R})$, one can prove using [23, Thm 11.6] that:

$$
Q_{y} \star u=P_{y} \star \mathcal{H} u=\mathcal{P}_{y}[\mathcal{H} u]
$$

Hence, from (3) and (4), we get that $Q_{y}=\mathcal{H} P_{y}$ on $L^{2}(\mathbb{R})$. We will also use in the sequel the following equality, for $g \in L^{2}(\mathbb{R})$ :

$$
\partial_{y} \mathcal{P}_{y}[g]=-\mathcal{H}\left(\partial_{x} \mathcal{P}_{y}[g]\right) .
$$

It can be obtained using the Fourier transform as in $[7,27]$. Actually, for $g \in L^{2}(\mathbb{R})$, $\partial_{y} \mathcal{P}_{y}[g]=-\left[\partial_{x} Q_{y} \star g\right]$, and $\partial_{x} \mathcal{P}_{y}[g]=\partial_{y} Q_{y} \star g$. And as a consequence of (5), and the isometric character of $\mathcal{H}$, we get $\left\|\partial_{y} \mathcal{P}_{y}[g]\right\|_{L^{2}(\mathbb{R})}=\left\|\partial_{x} \mathcal{P}_{y}[g]\right\|_{L^{2}(\mathbb{R})}$.

We also have the following uniqueness result, from [7, Lem. 2.19]:

Lemma 1 Let $h>0$ and $g \in L^{2}(\mathbb{R})$ such that $\partial_{x} \mathcal{P}_{h}[g]=0$ on a non-empty open subset of $\mathbb{R}$. Then $g \equiv 0$ on $\mathbb{R}$.

As a corollary to Lemma 1 , we get that if $g \in L^{2}(\mathbb{R})$ is such that $\mathcal{P}_{h}[g]=P_{h} \star g=0$ on a non-empty open subset of $\mathbb{R}$ for some $h>0$, then $g \equiv 0$ on $\mathbb{R}$.

\subsection{Hardy space $H^{2}$}

For $u \in L^{2}(\mathbb{R})$, the complex-valued function $P_{y} \star u+i Q_{y} \star u$ belongs to $H^{2}\left(\mathbb{C}_{+}\right)=H^{2}\left(\mathbb{C}_{+}, \mathbb{C}\right)$, the space of analytic functions $F$ on $\mathbb{C}_{+}$such that

$$
\|F\|_{H^{2}\left(\mathbb{C}_{+}\right)}:=\sup _{y>0}\left(\int_{-\infty}^{\infty}|F(x+i y)|^{2} d x\right)^{1 / 2}<\infty,
$$

see $[13,14]$. A function $F \in H^{2}\left(\mathbb{C}_{+}\right)$admits a non-tangential limit $F^{*} \in L^{2}(\mathbb{R}, \mathbb{C})$ as $y$ tends to 0 , almost everywhere on $\mathbb{R}$. The function $F^{*}$ belongs to the space $H^{2}(\mathbb{R})=H^{2}(\mathbb{R}, \mathbb{C})$ of functions $f \in L^{2}(\mathbb{R})$ such that

$$
\int_{-\infty}^{\infty} \frac{f(t)}{t-\bar{z}} d t=0, \quad z \in \mathbb{C}_{+} .
$$

The space $H^{2}(\mathbb{R})$ coincides with the space of boundary values on $\mathbb{R}$ (also "traces", if smoothness allows) of $H^{2}\left(\mathbb{C}_{+}\right)$functions. Indeed, the map $f \in H^{2}(\mathbb{R}) \longmapsto P_{y} \star f$ is an isometric isomorphism from $H^{2}(\mathbb{R})$ onto $H^{2}\left(\mathbb{C}_{+}\right)$.

It is not difficult to see that $(I+i \mathcal{H}) L^{2}(\mathbb{R})=H^{2}(\mathbb{R})$. As a consequence, the map $u \longmapsto$ 
$P_{y} \star(I+i \mathcal{H}) u$ is an isomorphism from $L^{2}(\mathbb{R})$ onto $H^{2}\left(\mathbb{C}_{+}\right)$. A function $f \in H^{2}\left(\mathbb{C}_{+}\right)$satisfies the boundary uniqueness Theorem [24, Cor. 6.4.2]: if $F \in H^{2}(\mathbb{R})$ is such that $F=0$ on $I \subset \mathbb{R}$ with $|I|>0$ then $F \equiv 0$ on $\mathbb{R}$.

Note that if $I \subset \mathbb{R}$, the space $(I+i \mathcal{H})\left(\widetilde{L^{2}(I)}\right)$ coincides with the space of functions $F^{*}$ of $H^{2}(\mathbb{R})$ such that supp Re $F^{*} \subset I$.

Observe that for $f \in L^{2}(\mathbb{R}, \mathbb{C})$,

$$
(I+i \mathcal{H}) f=2(I+i \mathcal{H})\left[\operatorname{Re} P_{+}(f)\right]=2 P_{+}(f),
$$

where $P_{+}(f)$ is the orthogonal projection from $L^{2}(\mathbb{R}, \mathbb{C})$ onto $H^{2}(\mathbb{R})$, see [21].

Finally, an amazing property of the Hilbert transform is given by the following Lemma (of which a proof can be found in [1, Lem. 2.12]):

Lemma 2 If $I, J \subset \mathbb{R}$ with $|I|,|J|>0$ such that $I \cap J=\emptyset$, then for any $g \in L^{2}(\mathbb{R})$ such that $\mathcal{H}\left(\chi_{J} g\right)=0$ on $I$, we have $g=0$ on $J$.

Lemma 2 can be seen as a consequence of the boundary uniqueness Theorem in $H^{2}(\mathbb{R})$. Indeed, if $\mathcal{H}\left(\chi_{J} g\right)=0$ on $I$ then the function in $H^{2}(\mathbb{R})$ given by $F=\chi_{J} g+i \mathcal{H}\left(\chi_{J} g\right)$ is equal to 0 on $I$ (as $J \cap I=\emptyset$ ). By the boundary uniqueness Theorem, it follows that $F=0$ on $\mathbb{R}$ and $g=0$ on $J$.

When $I \cap J \neq \emptyset$ and $I \nsubseteq I J$, Lemma 2 still holds true in some cases:

- $J \subsetneq I$ : indeed, the function $F=\chi_{J} g+i \mathcal{H}\left(\chi_{J} g\right)$ is equal to zero on $I \backslash J$ and the boundary uniqueness Theorem implies that $g=0$ on $J$, whenever $|I \backslash J|>0$;

- $I \cap J \neq \emptyset$ and $I \not \subset J$ : the same function $F$ is equal to zero on $I \backslash(I \cap J)$ and $g=0$ on $J$ by the boundary uniqueness Theorem, provided that $|I \backslash(I \cap J)|>0$.

However, if $I \subseteq J$ are bounded intervals, then there exists a function $g \in L^{2}(\mathbb{R}), g \neq 0$, such that $\mathcal{H}\left(\chi_{J} g\right) \neq 0$. With $I=(a, b)$, the (non-zero) function defined on $\mathbb{R}$ by $g(x)=$ $\chi_{I}(x) / \sqrt{(x-a)(b-x)}$ is such that $\mathcal{H}\left(\chi_{I} g\right)=\mathcal{H}(g)=0$ on $I$. Indeed, the function $x \mapsto$ $1 / \sqrt{(x-a)(b-x)}$ is shown in [19, Lem. 2.1] to be an eigenfunction of the operator $\mathcal{H}\left(\chi_{I} \cdot\right)_{\left.\right|_{I}}$ associated to the eigenvalue 0 .

- If $I=J$, the function $g$ furnishes a solution to the issue.

- If $I \subsetneq J$, one can write $\mathcal{H}\left(\chi_{J} g\right)=\mathcal{H}\left(\chi_{I} g\right)+\mathcal{H}\left(\chi_{J \backslash I} g\right)$. The above function $g$ vanishes outside $I$ hence on $J \backslash I$. Thus, $\mathcal{H}\left(\chi_{J \backslash I} g\right)=0$ on $\mathbb{R}$. Further, $\mathcal{H}\left(\chi_{I} g\right)=0$ on $I$, whence $\mathcal{H}\left(\chi_{J} g\right)=0$ on $I$, but $g \neq 0$ on $J$.

\section{Main operators}

Let $S$ and $K \subset \mathbb{R}$ be two nonempty open bounded intervals. Fix $h>0$. Let $\mathbf{m} \in L^{2}\left(S, \mathbb{R}^{2}\right)$. Taking the convolution of the PDE $\Delta U=\operatorname{div} \mathbf{m}$ by the fundamental solution to Laplace equation in dimension $n=2$ and applying Green formula, we obtain, for $X \in \mathbb{R}^{2} \backslash \operatorname{supp} \mathbf{m}$ :

$$
\begin{gathered}
U[\mathbf{m}](X)=-\frac{1}{2 \pi} \iint_{\mathbb{R}^{2}}\left(\operatorname{div}_{Y} \mathbf{m}\right)(Y) \log |X-Y| d Y=\frac{1}{2 \pi} \iint_{\mathbb{R}^{2}} \mathbf{m}(Y) \cdot \operatorname{grad}_{Y} \log |X-Y| d Y \\
=\frac{1}{2 \pi} \iint_{\mathbb{R}^{2}} \frac{\mathbf{m}(Y) \cdot(X-Y)}{|X-Y|^{2}} d Y .
\end{gathered}
$$

Since the support of $\mathbf{m}$ is a subset of $S \times\{0\}$, we get, at $X=(x, y) \in \mathbb{R}^{2} \backslash S \times\{0\}$, with $Y=(t, 0)$ :

$$
U[\mathbf{m}](x, y)=\frac{1}{2 \pi} \int_{S} \frac{m_{1}(t)(x-t)+m_{2}(t) y}{(x-t)^{2}+y^{2}} d t=\frac{1}{2}\left(Q_{y} \star \widetilde{m_{1}}+P_{y} \star \widetilde{m_{2}}\right)(x, y) .
$$


Let then $b_{2}[\mathbf{m}]=-\left(\partial_{y} U[\mathbf{m}]\right)_{\left.\right|_{K \times\{h\}}}$ (from now, we will ignore on the multiplicative constant $\left.\mu_{0} / 2\right)$ :

$$
\begin{aligned}
& b_{2}: \quad L^{2}\left(S, \mathbb{R}^{2}\right) \quad \longrightarrow L^{2}(K) \\
& \mathbf{m}=\left(m_{1}, m_{2}\right) \longmapsto-\partial_{y}\left(Q_{y} \star \widetilde{m_{1}}+P_{y} \star \widetilde{m_{2}}\right)_{\left.\right|_{K \times\{h\}}},
\end{aligned}
$$

with the use of Cauchy-Riemann equations, which also allow to get

$$
\begin{aligned}
b_{2}[\mathbf{m}] & =-\left(\partial_{x} \mathcal{P}_{y}\left[\widetilde{m_{1}}\right]+\partial_{y} \mathcal{P}_{y}\left[\widetilde{m_{2}}\right]\right)_{\left.\right|_{K \times\{h\}}}=-\left[\partial_{x}\left(P_{y} \star \widetilde{m_{1}}-Q_{y} \star \widetilde{m_{2}}\right)\right]_{\left.\right|_{K \times\{h\}}} \\
& =-\partial_{x} \mathcal{P}_{y}\left[\widetilde{m_{1}}-\mathcal{H} \widetilde{m_{2}}\right]_{\left.\right|_{K \times\{h\}}},
\end{aligned}
$$

where the second equality follows from (4). Using the boundedness of the convolution by $P_{y}$ from $L^{2}(\mathbb{R})$ to $W^{1,2}(\mathbb{R})$, the boundedness of the Hilbert transform and the equality $\left\|\widetilde{m_{i}}\right\|_{L^{2}(\mathbb{R})}=\left\|m_{i}\right\|_{L^{2}(S)}$, for $i \in\{1,2\}$ (see Section 2), one can prove that the operator $b_{2}$ is bounded $L^{2}\left(S, \mathbb{R}^{2}\right) \rightarrow L^{2}(Q)$.

Proposition 1 The adjoint operator $b_{2}^{*}$ to $b_{2}$ is given by

$$
\begin{aligned}
b_{2}^{*}: L^{2}(K) & \longrightarrow L^{2}\left(S, \mathbb{R}^{2}\right) \\
\phi & \longmapsto\left(\partial_{x}\left(P_{y} \star \widetilde{\phi}\right)_{\left.\right|_{S \times\{h\}}}, \partial_{x}\left(Q_{y} \star \widetilde{\phi}\right)_{\left.\right|_{S \times\{h\}}}\right)
\end{aligned}
$$

and is such that

$$
b_{2}^{*}[\phi]=\left(\left(\left(\partial_{x} P_{y}\right) \star \widetilde{\phi}\right)_{\left.\right|_{S \times\{h\}}},\left(\left(\partial_{x} Q_{y}\right) \star \widetilde{\phi}\right)_{\left.\right|_{S \times\{h\}}}\right)=\partial_{x}\left(P_{y} \star(I, \mathcal{H}) \widetilde{\phi}\right)_{\left.\right|_{S \times\{h\}}} .
$$

Proof: Let $y>0$ fixed. For $\mathbf{m} \in L^{2}\left(S, \mathbb{R}^{2}\right)$ and $\phi \in L^{2}(K)$, we have that

$$
\begin{aligned}
& \left\langle b_{2}[\mathbf{m}], \phi\right\rangle_{L^{2}(K)}=-\left\langle\partial_{x}\left(P_{y} \star \widetilde{m_{1}}\right), \phi\right\rangle_{L^{2}(K)}+\left\langle\partial_{x}\left(Q_{y} \star \widetilde{m_{2}}\right), \phi\right\rangle_{L^{2}(K)} \\
& =-\int_{\mathbb{R}} \int_{\mathbb{R}} \partial_{x}\left(P_{y}(x-t)\right) \widetilde{m_{1}}(t) \widetilde{\phi}(x) d t d x+\int_{\mathbb{R}} \int_{\mathbb{R}} \partial_{x}\left(Q_{y}(x-t)\right) \widetilde{m_{1}}(t) \widetilde{\phi}(x) d t d x \\
& =\int_{\mathbb{R}}\left(\int_{\mathbb{R}} \partial_{t}\left(P_{y}(x-t)\right) \widetilde{\phi}(x) d x\right) \widetilde{m_{1}}(t) d t+\int_{\mathbb{R}}\left(\int_{\mathbb{R}} \partial_{t}\left(Q_{y}(x-t)\right) \widetilde{\phi}(x) d x\right) \widetilde{m_{1}}(t) d t \\
& =\int_{S} \partial_{t}\left(\int_{\mathbb{R}} P_{y}(t-x) \widetilde{\phi}(x) d x\right) m_{1}(t) d t+\int_{S} \partial_{t}\left(\int_{\mathbb{R}} Q_{y}(t-x) \widetilde{\phi}(x) d x\right) m_{1}(t) d t \\
& =\left\langle m_{1}, \partial_{t}\left(P_{y} \star \widetilde{\phi}\right)\right\rangle_{L^{2}(S)}+\left\langle m_{2}, \partial_{t}\left(Q_{y} \star \widetilde{\phi}\right)\right\rangle_{L^{2}(S)} \\
& =\left\langle\left(m_{1}, m_{2}\right),\left(\partial_{t}\left(P_{y} \star \widetilde{\phi}\right), \partial_{t}\left(Q_{y} \star \widetilde{\phi}\right)\right)\right\rangle_{L^{2}\left(S, \mathbb{R}^{2}\right)},
\end{aligned}
$$

with

These operators have null kernel, as follows from the next results.

Proposition 2 The operator $b_{2}$ is injective.

Proof: Let $\mathbf{m} \in \operatorname{Ker} b_{2}$. Then, $\partial_{x} P_{y} \star\left(\widetilde{m_{1}}-\mathcal{H} \widetilde{m_{2}}\right)=0$ on $K \times\{h\}$, which implies that $\widetilde{m_{1}}-\mathcal{H} \widetilde{m_{2}}=0$ on $\mathbb{R}$ in view of Lemma 1 . Hence, $\widetilde{m_{1}}=\mathcal{H} \widetilde{m_{2}}$ which implies that $\mathcal{H} \widetilde{m_{2}}=0$ outside $S$ whence so does $\widetilde{m_{2}}+i \mathcal{H} \widetilde{m_{2}}$. Because it coincides with the boundary value of a function in $H^{2}\left(\mathbb{C}_{+}\right)$, namely $P_{y} \star\left(\widetilde{m_{2}}+i \mathcal{H} \widetilde{m_{2}}\right)$, this implies that $m_{2}=0$ (see $\left.[13,14]\right)$. Therefore, $m_{1}=0$ and Ker $b_{2}=\{0\}$.

In particular, contrarily to the situation in dimension 3 (and in higher dimensions) see [5], there are no non-vanishing "silent" sources (the corresponding so-called "null-space" is reduced to $\{0\})$.

Proposition 3 The operator $b_{2}^{*}$ is injective.

Proof: Let $f \in \operatorname{Ker} b_{2}^{*}$. Then, we have that $\partial_{x}\left(P_{y} \star(I, \mathcal{H}) \widetilde{f}\right)=(0,0)$ on $S \times\{h\}$ which implies that $\partial_{x}\left(P_{h} \star \widetilde{f}\right)=\partial_{x}\left(P_{h} \star \mathcal{H} \widetilde{f}\right)=0$ on $S$. By Lemma 1 , it follows that $\widetilde{f}=0$ on $\mathbb{R}$ and $f=0$ on $S$.

Moreover: 
Corollary 1 The operators $b_{2}$ and $b_{2}^{*}$ have a dense range.

Proof: Since $b_{2}^{*}$ is continuous (because so is $b_{2}$ ), the following orthogonal decomposition holds true:

$$
L^{2}\left(S, \mathbb{R}^{2}\right)=\operatorname{Ker} b_{2} \oplus \overline{\operatorname{Ran} b_{2}^{*}} .
$$

Hence, using Proposition $3, b_{2}^{*}$ has dense range in $L^{2}\left(S, \mathbb{R}^{2}\right)$. Similarly,

$$
L^{2}(K)=\operatorname{Ker} b_{2}^{*} \oplus \overline{\operatorname{Ran} b_{2}} .
$$

Hence, using Proposition 2, $b_{2}$ has dense range in $L^{2}(K)$.

Remark 1 Using the following equality for $f \in L^{2}(\mathbb{R}, \mathbb{C})$ :

$$
(I+i \mathcal{H}) f=(I+i \mathcal{H})(\operatorname{Re} f-\mathcal{H} \operatorname{Im} f) .
$$

with $\mathcal{H} u=\mathcal{H}\left[-i^{2} u\right]=-i \mathcal{H}[i u]$ whence $\mathcal{H}[i u]=i \mathcal{H} u$, we could consider the analytic versions of $b_{2}$ and $b_{2}^{*}$ denoted by $B_{2}$ and $B_{2}^{*}$ respectively (see [21]):

$$
\begin{aligned}
& B_{2}: L^{2}(S, \mathbb{C}) \quad \longrightarrow \quad L^{2}(K) \\
& m=m_{1}+i m_{2} \longmapsto-\operatorname{Re} \partial_{x}\left(P_{y} \star(I+i \mathcal{H}) \widetilde{m}\right)_{\left.\right|_{K \times\{h\}}}, \\
& B_{2}^{*}: L^{2}(K) \quad \longrightarrow \quad L^{2}(S, \mathbb{C}) \\
& \phi \quad \longmapsto \partial_{x}\left(P_{y} \star(I+i \mathcal{H}) \widetilde{\phi}\right)_{\mid S \times\{h\}} .
\end{aligned}
$$

Let $J$ be the isomorphism from $L^{2}(S, \mathbb{C})$ onto $L^{2}\left(S, \mathbb{R}^{2}\right)$ defined by $J\left(\phi_{1}+i \phi_{2}\right)=\left(\phi_{1}, \phi_{2}\right)$ which is unitary when $L^{2}(S, \mathbb{C})$ is equipped with the inner product $(1)$. We have that $B_{2}=$ $b_{2} \circ J$ and $b_{2}^{*}[\phi]=J \circ B_{2}^{*}[\phi], \phi \in L^{2}(K)$. As a consequence, $B_{2}$ and $B_{2}^{*}$ are injective and $\overline{\operatorname{Ran} B_{2}}=L^{2}(K), \overline{\operatorname{Ran} B_{2}^{*}}=L^{2}(S, \mathbb{C})$.

Proposition 4 The ranges of $b_{2}$ (respectively $B_{2}$ ) and $b_{2}^{*}$ (respectively $B_{2}^{*}$ ) are not closed: $\operatorname{Ranb}_{2} \subsetneq L^{2}(K), \operatorname{Ran}_{2}^{*} \subsetneq L^{2}\left(S, \mathbb{R}^{2}\right)$.

Proof: If we assume that the range of $B_{2}^{*}$ is closed, then Corollary 1 and Remark 1 are to the effect that $B_{2}^{*}$ is surjective. Hence, for $I \subsetneq S$, with $|I|>0$, there exists $\phi \in L^{2}(K)$ such that

$$
\partial_{x}\left(P_{h} \star(I+i \mathcal{H}) \widetilde{\phi}\right)_{S_{S}}=\chi_{I},
$$

It follows that $\partial_{x}\left(P_{h} \star(I+i \mathcal{H}) \widetilde{\phi}\right)=0$ on $S \backslash I$ whence $(I+i \mathcal{H}) \widetilde{\phi}=0$ on $\mathbb{R}$ by Lemma 1 . As in the proof of Proposition 2, this leads to $\phi=0$ which contradicts Equation (8). We conclude that $B_{2}^{*}$ is not surjective. As $b_{2}^{*}=J \circ B_{2}^{*}$, we get that $b_{2}^{*}$ is not surjective.

Now, if the range of $B_{2}$ is closed, then for any $g \in L^{2}(K)$, there would be $\phi \in L^{2}(S, \mathbb{C})$ such that

$$
g=-\operatorname{Re} \partial_{x}\left(P_{h} \star(I+i \mathcal{H}) \widetilde{\phi}\right)_{\left.\right|_{K \times\{h\}}} .
$$

Let $I \subsetneq K$ with $|I|>0$ and $g=\chi_{I}$. Then, there exists $\phi \in L^{2}(S, \mathbb{C})$ such that

$$
\operatorname{Re} \partial_{x}\left(P_{h} \star(I+i \mathcal{H}) \widetilde{\phi}\right)_{\left.\right|_{K}}=\partial_{x}\left(P_{h} \star \operatorname{Re}(I+i \mathcal{H}) \widetilde{\phi}\right)_{\left.\right|_{K}}=0 \quad \text { on } K \backslash I \text {. }
$$

By Lemma 1, we get that $\operatorname{Re}(I+i \mathcal{H}) \widetilde{\phi}=0$ on $\mathbb{R}$, and $\operatorname{Re} \widetilde{\phi}-\mathcal{H} \operatorname{Im} \widetilde{\phi}=0$ on $\mathbb{R}$. This means that $\mathcal{H} \operatorname{Im} \widetilde{\phi}=\operatorname{Re} \widetilde{\phi}$ on $\mathbb{R}$ whence $\mathcal{H} \operatorname{Im} \widetilde{\phi}=0$ on $\mathbb{R} \backslash S$, which proves that $\operatorname{Im} \widetilde{\phi}=0$ on $\mathbb{R}$ by Lemma 2 , and thus $\operatorname{Re} \widetilde{\phi}=0$ on $\mathbb{R}$. We conclude that $\phi=0$ on $\mathbb{R}$ which contradicts Equation (9). It follows that the range of $B_{2}$ is not closed and thus $b_{2}=J^{-1} \circ B_{2}$ is not surjective.

Remark 2 The ranges of $b_{2}\left(\right.$ and $B_{2}$ ) and $b_{2}^{*}$ (and $B_{2}^{*}$ ) are actually made of $C^{\infty}$-smooth functions on $K$ and $S$, respectively, see Section 2.2.

Proposition 5 The operators $b_{2}, B_{2}, b_{2}^{*}$ and $B_{2}^{*}$ are compact. 
Proof: Because $u \mapsto\left(\partial_{x} P_{y} \star u\right)_{\left.\right|_{S}}$ and $u \mapsto\left(\partial_{y} P_{y} \star u\right)_{\left.\right|_{S}}$ are compact operators on $\widetilde{\left.L^{2(K, \mathbb{C}}\right)}$ for $y>0$ (see Section 2.2), so is $B_{2}^{*}=\left(\partial_{x} \mathcal{P}_{h}(I+i H)\right)_{\left.\right|_{S}}$, and thus also $B_{2}$. As $b_{2}^{*}=J \circ B_{2}^{*}$ and $b_{2}=B_{2} \circ J^{-1}$, we get that $b_{2}$ and $b_{2}^{*}$ are compact.

Remark 3 We will also make use of the operator $a_{2}$ defined by

$$
\begin{array}{ccc}
a_{2}: L^{2}\left(S, \mathbb{R}^{2}\right) & \longrightarrow & L^{2}(K) \\
\mathbf{m}=\left(m_{1}, m_{2}\right) & \longmapsto & {\left[P_{y} \star\left(\widetilde{m_{1}}-\mathcal{H} \widetilde{m_{2}}\right)\right]_{\left.\right|_{K \times\{h\}}}}
\end{array},
$$

which is such that $b_{2}[\mathbf{m}]=-\partial_{x}\left(a_{2}[\mathbf{m}]\right)$.

We deduce from the proof of Proposition 1 that $a_{2}^{*}[\phi]=\left(P_{y} \star \widetilde{\phi}, P_{y} \star \mathcal{H} \widetilde{\phi}\right)_{\left.\right|_{S \times\{h\}}}$, for $\phi \in L^{2}(K)$, and from the one of Proposition 5 that $a_{2}$ and $a_{2}^{*}$ are Hilbert-Schmidt operators. Further, whenever $\phi \in W_{0}^{1,2}(K)$, then $b_{2}^{*}[\phi]=-a_{2}^{*}\left[\partial_{x} \phi\right]$.

\section{Bounded extremal problems for moments estimates}

We are interested in solving the following bounded extremal problem (BEP), with e $\in$ $L^{2}\left(S, \mathbb{R}^{2}\right)$ and $M \geq 0$ : find $\phi_{o} \in L^{2}(K),\left\|\phi_{o}\right\|_{L^{2}(K)} \leq M$ that satisfies

$$
\left\|b_{2}^{*}\left[\phi_{o}\right]-\mathbf{e}\right\|_{L^{2}\left(S, \mathbb{R}^{2}\right)}=\min _{\substack{\phi \in L^{2}(K) \\\|\phi\|_{L^{2}(K)} \leq M}}\left\|b_{2}^{*}[\phi]-\mathbf{e}\right\|_{L^{2}\left(S, \mathbb{R}^{2}\right)}
$$

Our motivation is as follows. Let $\mathbf{e}_{1}=\left(\chi_{S}, 0\right), \mathbf{e}_{2}=\left(0, \chi_{S}\right)$. A solution $\phi_{o}$ to $\left(\mathrm{BEP}_{m o}\right)$ furnishes a linear estimator in $L^{2}(K)$ for net moment estimates: indeed, for $i \in\{1,2\}$,

$$
\left|\left\langle b_{2}[\mathbf{m}], \phi_{i}\right\rangle_{L^{2}(K)}-\left\langle m_{i}\right\rangle\right|=\left|\left\langle\mathbf{m}, b_{2}^{*}\left[\phi_{i}\right]-\mathbf{e}_{i}\right\rangle_{L^{2}\left(S, \mathbb{R}^{2}\right)}\right| \leq\|\mathbf{m}\|_{L^{2}\left(S, \mathbb{R}^{2}\right)}\left\|b_{2}^{*}\left[\phi_{i}\right]-\mathbf{e}_{i}\right\|_{L^{2}\left(S, \mathbb{R}^{2}\right)},
$$

where $\phi_{i}=\phi_{o}\left[\mathbf{e}_{i}\right]$ is the solution of $\left(\mathrm{BEP}_{m o}\right)$ with $\mathbf{e}=\mathbf{e}_{i}$ and $\left\langle m_{i}\right\rangle=\int_{S} m_{i}(t) d t$.

Below, we consider BEP both in the Lebesgue space $L^{2}(K)$ and in the Sobolev space $W_{0}^{1,2}(K)$ in order to control the derivative and the oscillations of the solution.

\section{1 $\quad \mathrm{BEP}$ in $L^{2}(K)$}

\subsubsection{Well-posedness}

Proposition 6 Let $\mathbf{e} \in L^{2}\left(S, \mathbb{R}^{2}\right)$ and $M \geq 0$. Problem $\left(\mathrm{BEP}_{\text {mo }}\right)$ admits a unique solution $\phi_{o} \in L^{2}(K)$. Moreover, if $\mathbf{e} \notin$ Ran $b_{2}^{*}$, then $\phi_{o}$ saturates the constraint: $\left\|\phi_{o}\right\|_{L^{2}(K)}=M$.

Note that $\left\|\phi_{o}\right\|_{L^{2}(K)}=M$ also if $\mathbf{e}=b_{2}^{*}[\phi]$ for some $\phi \in L^{2}(K)$ such that $\|\phi\|_{L^{2}(K)} \geq M$.

Proof: Since $\operatorname{Ran} b_{2}^{*}$ is dense in $L^{2}\left(S, \mathbb{R}^{2}\right)$, the assumptions of [11, Lem. 2.1] (see also [12] and $\left[21\right.$, Lem. 1]) are satisfied, to the effect that if $M>0$, Problem $\left(\mathrm{BEP}_{m o}\right)$ admits a solution, which is unique and saturates the constraint if $\mathbf{e} \notin \operatorname{Ran} b_{2}^{*}$ (or if $\mathbf{e}=b_{2}^{*}[\phi]$ for $\phi \in L^{2}(K)$ such that $\left.\|\phi\|_{L^{2}(K)} \geq M\right)$. Observe further that because $b_{2}^{*}$ is injective (from Proposition 3), uniqueness still holds when $\mathbf{e} \in \operatorname{Ran} b_{2}^{*}$.

Existence and uniqueness could also be established by projection onto the closed convex set $\left[9\right.$, Thm 5.2] $b_{2}^{*}\left[B_{M}\right]$, for $B_{M}=\left\{\phi \in L^{2}(K),\|\phi\|_{L^{2}(K)} \leq M\right\}$. Indeed, $B_{M}$ is weakly compact as a ball in $L^{2}(K)$ and the weak closedness property of $b_{2}^{*}\left[B_{M}\right]$ follows from the continuity of $b_{2}^{*}$, see [6, Prop. 1]. By convexity of $B_{M}$ and continuity of $b_{2}^{*}, b_{2}^{*}\left[\phi_{n}\right]$ strongly converges to $b_{2}^{*}[\phi]$ in $L^{2}\left(S, \mathbb{R}^{2}\right)$ whenever $\phi_{n}$ weakly converges to $\phi$ in $L^{2}(K)$. Note that the compactness of $b_{2}^{*}$ is enough to get that $b_{2}^{*}\left[B_{M}\right]$ is closed. That the constraint is satisfied if $\mathbf{e} \notin b_{2}^{*}\left[B_{M}\right]$ could be established by differentiation of the squared criterion. 
Remark 4 The density property of $b_{2}^{*}\left[L^{2}(K)\right]$ in $L^{2}\left(S, \mathbb{R}^{2}\right)$ implies that without the norm constraint, Problem $\left(\mathrm{BEP}_{m o}\right)$ above is ill-posed. Indeed, if $\mathbf{e} \notin b_{2}^{*}\left[L^{2}(K)\right]$ the density gives the existence of a sequence $\left(\phi_{n}\right)$ of functions in $L^{2}(K)$ such that $\left\|b_{2}^{*}\left[\phi_{n}\right]-\mathbf{e}\right\|_{L^{2}\left(S, \mathbb{R}^{2}\right)} \rightarrow 0$. We claim that $\left\|\phi_{n}\right\|_{L^{2}(K)} \rightarrow \infty$ as $n \rightarrow \infty$. This can be seen by assuming $\left\|\phi_{n}\right\|_{L^{2}(K)}$ to remain bounded and extracting a weakly convergent subsequence, say $\left(\phi_{n}\right)$ again, to some $\phi \in b_{2}^{*}\left[B_{M}\right]$ (the later being weakly closed as we saw in the proof of Proposition 6). By convexity of $b_{2}^{*}\left[B_{M}\right]$, it follows that $\left(\phi_{n}\right)$ converges strongly to $\phi$ in $L^{2}\left(S, \mathbb{R}^{2}\right)$ and this implies that $\mathbf{e}=b_{2}^{*}[\phi] \in$ Ran $_{2}^{*}$ which leads to a contradiction.

\subsubsection{A constructive solution}

Assume that $\mathbf{e} \notin \operatorname{Ran} b_{2}^{*}$. From Proposition 6 , the solution of $\left(\mathrm{BEP}_{m o}\right)$ is a minimum on $L^{2}(K)$ of the functional $\phi \mapsto\left\|b_{2}^{*}[\phi]-\mathbf{e}\right\|_{L^{2}\left(S, \mathbb{R}^{2}\right)}^{2}$ which satisfies the constraint $\|\phi\|_{L^{2}(K)}^{2}=M^{2}$. Differentiating the criterion and the constraint w.r.t. $\phi$, we get that such a critical point $\phi_{o}$ is given by the equation:

$$
\left\langle b_{2} b_{2}^{*}\left[\phi_{o}\right], \psi\right\rangle_{L^{2}(K)}-\left\langle b_{2}[\mathbf{e}], \psi\right\rangle_{L^{2}(K)}=-\lambda\left\langle\phi_{o}, \psi\right\rangle_{L^{2}(K)},
$$

with $\gamma \in \mathbb{R}$ and for all $\psi \in L^{2}(K)$. It follows that:

$$
\left\langle b_{2} b_{2}^{*}\left[\phi_{o}\right]+\lambda \phi_{o}, \psi\right\rangle_{L^{2}(K)}=\left\langle b_{2}(\mathbf{e}), \psi\right\rangle_{L^{2}(K)} \text {, for all } \psi \in L^{2}(K),
$$

whence:

$$
b_{2} b_{2}^{*}\left[\phi_{o}\right]+\lambda \phi_{o}=b_{2}[\mathbf{e}] .
$$

We have $\lambda>0$. Indeed, taking $\psi=\phi_{o}$ in Equation (10), we get

$$
-\lambda\left\langle\phi_{o}, \phi_{o}\right\rangle_{L^{2}(K)}=-\lambda\left\|\phi_{o}\right\|_{L^{2}(K)}^{2}=\left\langle b_{2}^{*}\left(\phi_{o}\right)-\mathbf{e}, b_{2}^{*}\left(\phi_{o}\right)\right\rangle_{L^{2}\left(S, \mathbb{R}^{2}\right)} .
$$

By $\left[9\right.$, Thm 5.2], for any $\mathbf{g} \in L^{2}\left(S, \mathbb{R}^{2}\right)$,

$$
\operatorname{Re}\left\langle b_{2}^{*}\left(\phi_{o}\right)-\mathbf{e}, b_{2}^{*}\left(\phi_{o}\right)-\mathbf{g}\right\rangle_{L^{2}\left(S, \mathbb{R}^{2}\right)} \leq 0 .
$$

Taking $\mathbf{g}=0$, we obtain that $\left\langle b_{2}^{*}\left(\phi_{o}\right)-\mathbf{e}, b_{2}^{*}\left(\phi_{o}\right)\right\rangle_{L^{2}\left(S, \mathbb{R}^{2}\right)} \leq 0$. Thus, $-\lambda \leq 0$. As $\lambda=0$ if and only if $\mathbf{e} \in \operatorname{Ran} b_{2}^{*}$, we conclude that $-\lambda<0$.

Assume that $\mathbf{e} \notin \operatorname{Ran} b_{2}^{*}$. Then there exists a unique $\lambda>0$ such that $\left(\mathrm{CPE}_{m o}\right)$ holds true and $\left\|\phi_{o}\right\|_{L^{2}(K)}=M$. In this case,

$$
\lambda\left\|\phi_{o}\right\|_{L^{2}(K)}^{2}=\lambda M^{2}=-\left\langle b_{2}^{*}\left(\phi_{o}\right)-\mathbf{e}, b_{2}^{*}\left(\phi_{o}\right)\right\rangle_{L^{2}\left(S, \mathbb{R}^{2}\right)} .
$$

From now on, when there is no ambiguity, we will write $f^{\prime}=\partial_{x} f$ for the derivative of real-valued differentiable functions $f$ defined on $\mathbb{R}$ or on intervals of $\mathbb{R}$.

For $f \in L^{2}(K)$, at $y=h>0$, we have that:

$$
\begin{gathered}
b_{2} b_{2}^{*}[f]=\left[-P_{h} \star\left(\chi_{S}\left(P_{h} \star \widetilde{f}\right)^{\prime}\right)+Q_{h} \star\left(\chi_{S}\left(Q_{h}^{\prime} \star \widetilde{f}\right)\right)\right]_{\left.\right|_{K}}^{\prime} \\
=\left[-P_{h}^{\prime} \star\left(\chi_{S}\left(P_{h}^{\prime} \star \widetilde{f}\right)\right)+Q_{h}^{\prime} \star\left(\chi_{S}\left(Q_{h}^{\prime} \star \widetilde{f}\right)\right)\right]_{\left.\right|_{K}} .
\end{gathered}
$$

The critical point equation $\left(\mathrm{CPE}_{m o}\right)$ can be written as:

$$
\left[-P_{h}^{\prime} \star\left(\chi_{S}\left(P_{h}^{\prime} \star \widetilde{\phi_{o}}\right)\right)+Q_{h}^{\prime} \star\left(\chi_{S}\left(Q_{h}^{\prime} \star \widetilde{\phi_{o}}\right)\right)\right]_{\left.\right|_{K}}+\lambda \phi_{o}=-P_{h}^{\prime} \star\left(\widetilde{e_{1}}-\mathcal{H} \widetilde{e_{2}}\right)_{\left.\right|_{K}},
$$

where $\left(e_{1}, e_{2}\right)=\mathbf{e}$. 
Observe that, if the function $\eta \in L^{2}(K)$ represents the error on the measurements of $b_{2}[\mathbf{m}]$, then

$$
\begin{aligned}
\left|\left\langle b_{2}[\mathbf{m}]+\eta, \phi_{i}\right\rangle_{L^{2}(K)}-\left\langle m_{i}\right\rangle\right| & =\left|\left\langle\mathbf{m}, b_{2}^{*}\left[\phi_{i}\right]-\mathbf{e}_{i}\right\rangle_{L^{2}\left(S, \mathbb{R}^{2}\right)}+\left\langle\eta, \phi_{i}\right\rangle_{L^{2}(K)}\right| \\
& \leq\|\mathbf{m}\|_{L^{2}\left(S, \mathbb{R}^{2}\right)}\left\|b_{2}^{*}\left[\phi_{i}\right]-\mathbf{e}_{i}\right\|_{L^{2}\left(S, \mathbb{R}^{2}\right)}+\|\eta\|_{L^{2}(K)}\left\|\phi_{i}\right\|_{L^{2}(K)} \\
& \leq\|\mathbf{m}\|_{L^{2}\left(S, \mathbb{R}^{2}\right)}\left\|b_{2}^{*}\left[\phi_{i}\right]-\mathbf{e}_{i}\right\|_{L^{2}\left(S, \mathbb{R}^{2}\right)}+M\|\eta\|_{L^{2}(K)} .
\end{aligned}
$$

For a choice of $M>0$, the solution of $\left(\mathrm{BEP}_{m o}\right)$ guarantees that $\left\|b_{2}^{*}\left[\phi_{i}\right]-\mathbf{e}_{i}\right\|_{L^{2}\left(S, \mathbb{R}^{2}\right)}$ is minimal and the term $\|\eta\|_{L^{2}(K)}$ is controlled by $M=\left\|\phi_{i}\right\|_{L^{2}(K)}$. The challenge of the numerical implementations is to choose a right $M>0$ such that $\phi_{i}$ satisfies the two conditions mentioned above. We will observe in numerical implementations that the solution $\phi_{i}$ can oscillate at the endpoints. However, since $\operatorname{Ran} b_{2} \subset W^{1,2}(K)$, then $\left(\mathrm{CPE}_{m o}\right)$ is to the effect that $\phi_{i} \in W^{1,2}(K) \subset C(\bar{K})$.

\section{2 $\quad$ BEP in $W_{0}^{1,2}(K)$}

An alternative is to formulate the BEP in a space of functions such that these oscillations are controlled. It thus seems natural to search for a solution in the Sobolev space $W_{0}^{1,2}(K)$. We denote by $b_{\left.2\right|_{W}}^{*}$ the restriction of $b_{2}^{*}$ to $W_{0}^{1,2}(K)$. For $\phi \in W_{0}^{1,2}(K)$,

$b_{2}^{*}[\phi]=b_{\left.2\right|_{W}}^{*}[\phi]=\left(\left(P_{h} \star \widetilde{\phi}\right)_{\left.\right|_{S}}^{\prime},\left(Q_{h} \star \widetilde{\phi}\right)_{\left.\right|_{S}}^{\prime}\right)=\left(\left(P_{h}^{\prime} \star \widetilde{\phi}\right)_{\left.\right|_{S}},\left(Q_{h}^{\prime} \star \widetilde{\phi}\right)_{\left.\right|_{S}}\right)=\left(\left(P_{h} \star \widetilde{\phi^{\prime}}\right)_{\left.\right|_{S}},\left(Q_{h} \star \widetilde{\phi^{\prime}}\right)_{\left.\right|_{S}}\right)$,

where $(\widetilde{\phi})^{\prime}=\widetilde{\phi}^{\prime}=\phi^{\prime} \vee 0$. Notice that $\widetilde{\phi} \in W^{1,2}(\mathbb{R})$ only when $\phi \in W_{0}^{1,2}(K)$ (see [9]).

Let $\mathbf{e} \in L^{2}\left(S, \mathbb{R}^{2}\right)$ and $M \geq 0$. The problem can thus be stated as the one of finding $\phi_{o} \in W_{0}^{1,2}(K),\left\|\phi_{o}\right\|_{W_{0}^{1,2}(K)} \leq M$ such that:

$$
\left\|b_{\left.2\right|_{W}}^{*}\left[\phi_{o}\right]-\mathbf{e}\right\|_{L^{2}\left(S, \mathbb{R}^{2}\right)}=\min _{\substack{\phi \in W_{0}^{1,2}(K) \\\|\phi\|_{W_{0}^{1,2}(K)}^{1,2} \leq M}}\left\|b_{\left.2\right|_{W}}^{*}[\phi]-\mathbf{e}\right\|_{L^{2}\left(S, \mathbb{R}^{2}\right)} \cdot \quad\left(\mathrm{BEP}_{m o, W}\right)
$$

\subsubsection{Well-posedness}

Proposition 7 Let $\mathbf{e} \in L^{2}\left(S, \mathbb{R}^{2}\right)$ and $M \geq 0$. Problem $\left(\mathrm{BEP}_{m o, W}\right)$ admits a unique solution $\phi \in W_{0}^{1,2}(K)$. If $\mathbf{e} \notin b_{\left.2\right|_{W} ^{*}}^{*}\left[W_{0}^{1,2}(K)\right]$, then $\phi_{o}$ saturates the constraint: $\left\|\phi_{o}\right\|_{W_{0}^{1,2}(K)}=$ $M$.

Proof: We apply again [11, Lemma 2.1] in order to get existence and uniqueness of a solution $\phi_{o} \in W_{0}^{1,2}(K)$ to $\left(\mathrm{BEP}_{m o, W}\right)$ saturating the constraint if $\mathbf{e} \notin b_{\left.2\right|_{W}}^{*}\left[W_{0}^{1,2}(K)\right]$. Yet, because $b_{2}^{*}$ is injective (from Proposition 3), uniqueness still holds when e $\in b_{\left.2\right|_{W}}^{*}\left[W_{0}^{1,2}(K)\right]$. Note that in this case, the constraint is saturated also if $\mathbf{e}=b_{2}^{*}[\phi]$ for $\phi \in L^{2}(K)$ such that $\|\phi\|_{W_{0}^{1,2}(K)} \geq M$.

Remark 5 Let $\psi \in L^{2}(K)$ with zero mean on $K$. There exists a unique $\phi \in W_{0}^{1,2}(K)$ such that $\phi^{\prime}=\psi$ (and $\left.\|\phi\|_{W_{0}^{1,2}(K)}=\left\|\phi^{\prime}\right\|_{L^{2}(K)}\right)$. Then, $\left(\mathrm{BEP}_{m o, W}\right)$ is equivalent to the following problem: let $\mathbf{e} \in L^{2}\left(S, \mathbb{R}^{2}\right)$ and $M \geq 0$, find $\psi_{o} \in L^{2}(K)$ such that $\int_{K} \psi_{o}(x) d x=0$, $\left\|\psi_{o}\right\|_{L^{2}(K)} \leq M$ that satisfies

$$
\left\|a_{2}^{*}\left[\widetilde{\psi_{o}}\right]-\mathbf{e}\right\|_{L^{2}\left(S, \mathbb{R}^{2}\right)}=\min _{\substack{\psi \in L^{2}(K) \\ S_{K} \psi(x) d x=0,\|\psi\|_{L^{2}(K)} \leq M}}\left\|a_{2}^{*}[\widetilde{\psi}]-\mathbf{e}\right\|_{L^{2}\left(S, \mathbb{R}^{2}\right)}
$$

Proposition 7 is to the effect that a unique solution $\phi_{o}$ to $\left(\mathrm{BEP}_{m o, W}\right)$ does exist, whence also a unique solution $\psi_{o}$ to (11), which is such that $\phi_{o}^{\prime}=\psi_{o}$.

We also have the following density result.

Lemma $3 b_{2}^{*}\left[W_{0}^{1,2}(K)\right]$ is dense in $L^{2}\left(S, \mathbb{R}^{2}\right)$. 
Proof: First, observe that $W_{0}^{1,2}(K)$ is dense in $L^{2}(K)$ since the set $C_{c}^{\infty}(K)$ of $C^{\infty}(K)$ functions with compact support $\subset K$ is dense in $L^{2}(K)$ (see [9, Cor. 4.23]). As $W^{1,2}(K) \subset$ $L^{2}(K)$, we use the continuity of $b_{2}^{*}$ to get:

$$
b_{2}^{*}\left[W_{0}^{1,2}(K)\right] \subset b_{2}^{*}\left(L^{2}(K)\right)=b_{2}^{*}\left[{\overline{W_{0}^{1,2}(K)}}^{L^{2}(K)}\right] \subset{\overline{b_{2}^{*}\left[W_{0}^{1,2}(K)\right]}}^{L^{2}\left(S, \mathbb{R}^{2}\right)},
$$

Recall that $b_{2}^{*}\left[L^{2}(K)\right]$ is dense in $L^{2}\left(S, \mathbb{R}^{2}\right)$ (for the $L^{2}\left(S, \mathbb{R}^{2}\right)$ norm), from Corollary 1 . We therefore conclude to the density of $b_{2}^{*}\left[W_{0}^{1,2}(K)\right]$ in $L^{2}\left(S, \mathbb{R}^{2}\right)$.

Yet, Lemma 3 implies that without the norm constraint, Problem $\left(\mathrm{BEP}_{m o, W}\right)$ is ill-posed. The minimum is in this case an infimum and is equal to 0 while minimizing sequences diverge.

\subsubsection{A constructive solution}

Observe that $b_{2}=\left(\left.b_{2}^{*}\right|_{W}\right)^{*}$, since $b_{\left.2\right|_{W}}^{*}=a_{2}^{*}\left(\phi^{\prime}\right)$, where $\phi^{\prime}$ denotes the derivation from $W_{0}^{1,2}(K)$ onto the space of $L^{2}(K)$-functions with zero mean. Thus, for $f \in W_{0}^{1,2}(K)$, we get

$$
b_{2} b_{\left.2\right|_{W}}^{*}[f]=\left[-P_{h}^{\prime} \star\left(\chi_{S} P_{h} \star \tilde{f}^{\prime}\right)+Q_{h}^{\prime} \star\left(\chi_{S} Q_{h} \star \tilde{f}^{\prime}\right)\right]_{\left.\right|_{K}} .
$$

Assume that $\mathbf{e} \notin b_{\left.2\right|_{W}}^{*}\left[W_{0}^{1,2}(K)\right]$. From Proposition 7 , we obtain a critical point equation somewhat different from $\left(\mathrm{CPE}_{m o}\right)$ for the solution $\phi_{o}$ in this case (see also Section 4.1.2):

$$
\left\langle b_{2} b_{\left.2\right|_{W}}^{*}\left[\phi_{o}\right], \psi\right\rangle_{L^{2}(K)}-\left\langle b_{2}[\mathbf{e}], \psi\right\rangle_{L^{2}(K)}=\gamma\left\langle\phi_{o}^{\prime}, \psi^{\prime}\right\rangle_{L^{2}(K)}=-\gamma\left\langle\phi_{o}^{\prime \prime}, \psi\right\rangle_{L^{2}(K)},
$$

with $\gamma<0$ and $\forall \psi \in W_{0}^{1,2}(K)$. The solution $\phi_{o} \in W_{0}^{1,2}(K)$ of $\left(\mathrm{BEP}_{m o, W}\right)$ is thus given by

$$
b_{2} b_{\left.2\right|_{W}}^{*}\left[\phi_{o}\right]+\gamma \phi_{o}^{\prime \prime}=b_{2}[\mathbf{e}] \text {, }
$$

$\left(\mathrm{CPE}_{m o, W}\right)$

with $\gamma<0$, such that $\left\|\phi_{o}\right\|_{W^{1,2}(K)}=\left\|\phi_{o}^{\prime}\right\|_{L^{2}(K)}=M$ if $\mathbf{e} \notin \operatorname{Ran} b_{\left.2\right|_{W}}^{*}$. The equation $\mathrm{CPE}_{m o, W}$ is considered in the weak sense in $L^{2}(K)$. Thus,

$$
\left[-P_{h}^{\prime} \star\left(\chi_{S}\left(P_{h} \star \widetilde{\phi_{o}^{\prime}}\right)\right)+Q_{h}^{\prime} \star\left(\chi_{S}\left(Q_{h} \star \widetilde{\phi_{o}^{\prime}}\right)\right)\right]_{\left.\right|_{K}}+\gamma \phi_{o}^{\prime \prime}=-P_{h}^{\prime} \star\left(\widetilde{e_{1}}-\mathcal{H} \widetilde{e_{2}}\right)_{\left.\right|_{K}}
$$

\subsubsection{An equivalent problem in $H^{2}$}

Let $\mathbb{C}_{+}=\{x+i y \in \mathbb{C}: x \in \mathbb{R}, y>0\}$ and $H^{2}\left(\mathbb{C}_{+}\right)$denotes the Hardy space of the upper half-plane defined in Section 2.3. As a function $F \in H^{2}\left(\mathbb{C}_{+}\right)$can be identified to its boundary function $F^{*} \in H^{2}(\mathbb{R})$ so that $F_{\left.\right|_{S \times\{0\}}}$ can be defined as $F_{\left.\right|_{S}}^{*}$.

Given a function $g \in L^{2}(S, \mathbb{C})$, for $\varepsilon>0$, there is a function $F \in H^{2}\left(\mathbb{C}_{+}\right)$such that $\left\|g-F_{\left.\right|_{S}}^{*}\right\|_{L^{2}(S, \mathbb{C})}<\varepsilon$. Indeed, if $f \in\left(H^{2}(\mathbb{R})_{\left.\right|_{S}}\right)^{\perp}$, then for $g \in H^{2}(\mathbb{R})$,

$$
\left\langle f, g_{\left.\right|_{S}}\right\rangle_{L^{2}(S, \mathbb{C})}=\langle\tilde{f}, g\rangle_{L^{2}(\mathbb{R}, \mathbb{C})}=0 .
$$

Taking $g(t)=\frac{1}{t-z}$ with $z \in \mathbb{C}$ such that $\operatorname{Im} z>0$, it follows that

$$
\int_{-\infty}^{+\infty} \frac{\tilde{f}(t)}{t-\bar{z}}=0, \quad z \in \mathbb{C}_{+}
$$

and $\tilde{f} \in H_{-}^{2}(\mathbb{R})$. By the boundary uniqueness Theorem, $f=0$ on $S$ so $H^{2}(\mathbb{R})_{\left.\right|_{S}}$ is dense in $L^{2}(S, \mathbb{C})$.

For $M \geq 0$, let

$$
\mathcal{B}_{M}=\left\{F \in H^{2}\left(\mathbb{C}_{+}\right): \operatorname{supp} \operatorname{Re} F^{*} \subset K \text { and }\left\|\operatorname{Re} F^{*}\right\|_{L^{2}(K)} \leq M\right\} \subset H^{2}\left(\mathbb{C}_{+}\right) .
$$


Proposition 8 Let $g \in L^{2}(S, \mathbb{C})$. The problem $\left(\mathrm{BEP}_{m o, W}\right)$ is equivalent to the following bounded extremal problem (with $L^{2}(S, \mathbb{C})$ norm):

$$
\min _{F \in \mathcal{B}_{M}}\left\|F(.+i h)_{\left.\right|_{S}}-g\right\|_{L^{2}(S, \mathbb{C})}, .
$$

Proof: Let $\mathbf{e} \in L^{2}\left(S, \mathbb{R}^{2}\right)$ be such that $J(g)=\mathbf{e}$. By Proposition 7 , there exists a function $\phi_{o} \in W_{0}^{1,2}(K)$ such that

$$
\left\|b_{\left.2\right|_{W}}^{*}\left[\phi_{o}\right]-\mathbf{e}\right\|_{L^{2}\left(S, \mathbb{R}^{2}\right)}=\min _{\substack{\phi \in W_{0}^{1,2}(K) \\ \| \phi W_{0}^{1,2}(K)}}\left\|b_{\left.2\right|_{W}}^{*}[\phi]-\mathbf{e}\right\|_{L^{2}\left(S, \mathbb{R}^{2}\right)} .
$$

Using the isomorphism $J$, we get for any $\phi \in W_{0}^{1,2}(K)$,

$$
\left\|b_{\left.2\right|_{W}}^{*}[\phi]-\mathbf{e}\right\|_{\left(L^{2}\left(S, \mathbb{R}^{2}\right)\right.}=\left\|J\left(B_{\left.2\right|_{W}}^{*}[\phi]\right)-\mathbf{e}\right\|_{L^{2}\left(S, \mathbb{R}^{2}\right)}=\left\|B_{\left.2\right|_{W}}^{*}[\phi]-J^{-1} \mathbf{e}\right\|_{L^{2}(S, \mathbb{C})},
$$

where $B_{\left.2\right|_{W}}^{*}[\phi]=\left(P_{h} \star(I+i \mathcal{H}) \widetilde{\psi}\right)_{\left.\right|_{S}}$ and $\psi$ is the (unique) function of $L^{2}(K)$ such that $\phi^{\prime}=\psi$. Let $F_{o} \in \mathcal{B}_{M}$ defined by $P_{y} \star(I+i \mathcal{H}) \widetilde{\psi_{o}}=F_{o}$ with $\phi_{o}^{\prime}=\psi_{o}$. It follows that $F_{o}(.+i h)=B_{\left.2\right|_{W}}^{*}\left[\phi_{o}\right]=\left(P_{h} \star(I+i \mathcal{H}) \widetilde{\psi_{o}}\right)$ and $\left(\operatorname{Re} F_{o}^{*}\right)_{\left.\right|_{K}}=\psi_{o}$. Then,

$$
\begin{aligned}
\left\|F_{o}(.+i h)_{\left.\right|_{S}}-g\right\|_{L^{2}(S, \mathbb{C})} & =\left\|B_{\left.2\right|_{W}}^{*}\left[\phi_{o}\right]-J^{-1} \mathbf{e}\right\|_{L^{2}(S, \mathbb{C})} \\
& =\min _{\substack{\phi \in W_{0}^{1,2}(K) \\
\|\phi\|_{W^{1,2}(K)} \leq M}}\left\|B_{\left.2\right|_{W}}^{*}[\phi]-J^{-1} \mathbf{e}\right\|_{L^{2}\left(S, \mathbb{R}^{2}\right)} \\
& =\min _{\substack{\phi \in W_{0}^{1,2}(K) \\
\|\phi\|_{W^{1,2}(K)} \leq M}}\left\|\left.\left(P_{h} \star(I+i \mathcal{H}) \widetilde{\phi^{\prime}}\right)\right|_{S_{S}}-J^{-1} \mathbf{e}\right\|_{L^{2}\left(S, \mathbb{R}^{2}\right)} \\
& =\min _{\substack{\psi \in L^{2}(K) \\
\|\psi\|_{L^{2}(K)} \leq M}}\left\|\left(P_{h} \star(I+i \mathcal{H}) \widetilde{\psi}\right)_{\left.\right|_{S}}-J^{-1} \mathbf{e}\right\|_{L^{2}\left(S, \mathbb{R}^{2}\right)} \\
& =\min _{F \in \mathcal{B}_{M}}\left\|F(.+i h)_{\left.\right|_{S}}-g\right\|_{L^{2}(S, \mathbb{C})},
\end{aligned}
$$

where we use that $P_{y} \star(I+i \mathcal{H}) \widetilde{\psi} \in \mathcal{B}_{M}$ for any $\psi \in L^{2}(K)$.

\section{Algorithms and numerical computations}

\subsection{Construction of the algorithms}

\subsubsection{Bases functions}

Let $S=(-s, s), K=(-q, q), s, q \in \mathbb{R}_{+}$. For $n \in \mathbb{Z}$, let $g_{n}(x)=\exp (i n \pi x / q)$, for $x \in K$. They are eigenfunctions of the Laplacian on $K$ :

$$
g_{n}^{\prime \prime}=-\left[\frac{n \pi}{q}\right]^{2} g_{n}=-\mu_{n} g_{n}, \quad \text { where } \quad \mu_{n}=\left[\frac{n \pi}{q}\right]^{2}>0 .
$$

Up to multiplication by the constant factor $\frac{1}{(2 q)^{1 / 2}}$ for normalization, the family of functions $\left(g_{n}\right)_{n}, n \in \mathbb{Z}$, is the Hilbertian Fourier basis of $L^{2}(K, \mathbb{C})$ (see $[9$, Sec. 8.6]). We use it to expand and compute the solutions to the above bounded extremal problems, $\left(\mathrm{BEP}_{m o}\right)$ in $L^{2}(K)$ and $\left(\mathrm{BEP}_{m o, W}\right)$ in $W_{0}^{1,2}(K)$. Indeed, functions $\phi$ in $L^{2}(K)$ can be expanded in Fourier series on the basis $\left(g_{n}\right)_{n}, n \in \mathbb{Z}$ :

$$
\phi=\sum_{n \in \mathbb{Z}} c_{n} g_{n}
$$

with $c_{n} \in l^{2}(\mathbb{Z}), c_{-n}=\bar{c}_{n}, c_{0} \in \mathbb{R}$ (since $\phi$ is real-valued). Moreover, $\phi$ belongs to $W^{1,2}(K)$ if, and only if, the coefficients of its expansion on $\left(g_{n}\right)_{n}, n \in \mathbb{Z}$, are such that $n c_{n} \in l^{2}(\mathbb{Z})$ and $c_{n}=-c_{-n} \in i \mathbb{R}$. 
Another family of appropriate functions in $L^{2}(K)$ is made of piecewise constant functions on small intervals (say $\left.K_{l}, l=1, \cdots, L\right)$ covering $K=(-q, q)$ (so called $P_{0}$ finite elements), see also [6]. We use it for the computations of the solutions to the associated forward problem (Section 5.1.3).

\subsubsection{Operators, matrices in $L^{2}(K)$ and $W_{0}^{1,2}(K)$}

For all $\phi \in L^{2}(K)$, we use the expression

$$
b_{2}^{*}[\phi]=\left(\left(\partial_{x} P_{y}\right) \star \widetilde{\phi},\left(\partial_{x} Q_{y}\right) \star \widetilde{\phi}\right)_{\left.\right|_{S \times\{h\}}}=\left(P_{y}^{\prime} \star \widetilde{\phi}, Q_{y}^{\prime} \star \widetilde{\phi}\right)_{\left.\right|_{S \times\{h\}}} .
$$

Solutions to $\left(\mathrm{BEP}_{m o}\right)$ in $L^{2}(K)$ (actually in $\left.W^{1,2}(K) \subset L^{2}(K)\right)$ are provided by the critical point equation $\left(\mathrm{CPE}_{m o}\right)$.

For moments estimation, we consider the specific functions $\mathbf{e}=\mathbf{e}_{1}, \mathbf{e}_{2}$, with $\mathbf{e}_{1}=\left(\chi_{S}, 0\right)$ for $\left\langle m_{1}\right\rangle, \mathbf{e}_{2}=\left(0, \chi_{S}\right)$ for $\left\langle m_{2}\right\rangle$. From Lemma 1 and the relation (4), it holds that $\mathbf{e}_{i} \notin \operatorname{Ran} b_{2}^{*}$. Hence, the constraint in the BEP is saturated by the approximant, following Propositions 6 and 7.

Hence, using the Fourier basis $\left(g_{n}\right)$ in order to express solutions to $\left(\mathrm{CPE}_{m o}\right)$ as $\phi_{o}=$ $\sum_{n \in \mathbb{Z}} c_{n} g_{n}$, we get that, $\forall k \in \mathbb{Z}$ :

$$
\sum_{n \in \mathbb{Z}} c_{n}\left\langle b_{2}^{*}\left[g_{n}\right], b_{2}^{*}\left[g_{k}\right]\right\rangle_{L^{2}\left(S, \mathbb{R}^{2}\right)}+\lambda c_{k}=\left\langle\mathbf{e}, b_{2}^{*}\left[g_{k}\right]\right\rangle_{L^{2}\left(S, \mathbb{R}^{2}\right)},
$$

for $\lambda>0$ such that $\left\|\phi_{o}\right\|_{L^{2}(K)}^{2}=\sum_{n \in \mathbb{Z}}\left|c_{n}\right|^{2}=M^{2}$.

For $t_{1}, t_{2} \in K, x \in S$, let:

$$
I\left(t_{1}, t_{2}, x\right)=P_{h}^{\prime}\left(x-t_{1}\right) P_{h}^{\prime}\left(x-t_{2}\right)+Q_{h}^{\prime}\left(x-t_{1}\right) Q_{h}^{\prime}\left(x-t_{2}\right),
$$

and

$$
k\left(t_{1}, t_{2}\right)=\int_{-s}^{s} I\left(t_{1}, t_{2}, x\right) d x .
$$

Following the expressions of the operator $b_{2}^{*}$ in Section 3, we obtain:

\section{Lemma 4}

$$
\left\langle b_{2}^{*}\left[g_{n}\right], b_{2}^{*}\left[g_{k}\right]\right\rangle_{L^{2}\left(S, \mathbb{R}^{2}\right)}=\frac{1}{\pi^{2}} \int_{-q}^{q} \int_{-q}^{q} k\left(t_{1}, t_{2}\right) e^{i \frac{\pi}{q}\left(n t_{1}+k t_{2}\right)} d t_{1} d t_{2} .
$$

These are the Fourier coefficients of $\chi_{K}\left(t_{1}\right) \chi_{K}\left(t_{2}\right) k\left(t_{1}, t_{2}\right)$.

Proof: Observe that

$$
\begin{aligned}
&\left\langle b_{2}^{*}[\phi], b_{2}^{*}[\psi]\right\rangle_{L^{2}\left(S, \mathbb{R}^{2}\right)}=\left\langle P_{h}^{\prime} \star \widetilde{\phi}, P_{h}^{\prime} \star \widetilde{\psi}\right\rangle_{L^{2}(S)}+\left\langle Q_{h}^{\prime} \star \widetilde{\phi}, Q_{h}^{\prime} \star \widetilde{\psi}\right\rangle_{L^{2}(S)} \\
&=\int_{-s}^{s} P_{h}^{\prime} \star \widetilde{\phi}(x) P_{h}^{\prime} \star \widetilde{\psi}(x) d x+\int_{-s}^{s} Q_{h}^{\prime} \star \widetilde{\phi}(x) Q_{h}^{\prime} \star \widetilde{\psi}(x) d x \\
&=\int_{-s}^{s} \int_{-q}^{q} \int_{-q}^{q}\left(P_{h}^{\prime}\left(x-t_{1}\right) P_{h}^{\prime}\left(x-t_{2}\right)+Q_{h}^{\prime}\left(x-t_{1}\right) Q_{h}^{\prime}\left(x-t_{2}\right)\right) \phi\left(t_{1}\right) \psi\left(t_{2}\right) d t_{1} d t_{2} d x .
\end{aligned}
$$

We have, for $\phi, \psi \in L^{2}(K)$,

$$
\left\langle b_{2}^{*}[\phi], b_{2}^{*}[\psi]\right\rangle_{L^{2}\left(S, \mathbb{R}^{2}\right)}=\frac{1}{\pi^{2}} \int_{-s}^{s} \int_{-q}^{q} \int_{-q}^{q} I\left(t_{1}, t_{2}, x\right) \phi\left(t_{1}\right) \psi\left(t_{2}\right) d t_{1} d t_{2} d x .
$$

We can interchange the integrals, which leads to

$$
\left\langle b_{2}^{*}[\phi], b_{2}^{*}[\psi]\right\rangle_{L^{2}\left(S, \mathbb{R}^{2}\right)}=\frac{1}{\pi^{2}} \int_{-q}^{q} \int_{-q}^{q} k\left(t_{1}, t_{2}\right) \phi\left(t_{1}\right) \psi\left(t_{2}\right) d t_{1} d t_{2} .
$$


Taking $\phi=g_{n}$ and $\psi=g_{k}$ leads to the expression (13).

This allows to compute the left-hand side of $\left(\mathrm{CPE}_{m o}\right)$ and of (12).

Similarly, solutions to $\left(\mathrm{BEP}_{m o, W}\right)$ in $W_{0}^{1,2}(K)$, are furnished by $\left(\mathrm{CPE}_{m o, W}\right)$. Notice that $b_{\left.2\right|_{W}}^{*}[\phi]=b_{2}^{*}[\phi]$ if $\phi \in W_{0}^{1,2}(K)$. Using $\left(g_{n}\right)$ in order to express the solutions to $\left(\mathrm{CPE}_{m o, W}\right)$ as $\phi_{o}=\sum_{n \in \mathbb{Z}} d_{n} g_{n}$, we get that, $\forall k \in \mathbb{Z}$ :

$$
\sum_{n \in \mathbb{Z}} d_{n}\left\langle b_{2}^{*}\left[g_{n}\right], b_{2}^{*}\left[g_{k}\right]\right\rangle_{L^{2}\left(S, \mathbb{R}^{2}\right)}-\gamma \mu_{k} d_{k}=\left\langle\mathbf{e}, b_{2}^{*}\left[g_{k}\right]\right\rangle_{L^{2}\left(S, \mathbb{R}^{2}\right)}
$$

for $\gamma<0$ such that $\left\|\phi_{o}^{\prime}\right\|_{L^{2}(K)}^{2}=\sum_{n \in \mathbb{Z}} \mu_{n}\left|d_{n}\right|^{2}=M^{2}$, which has to be compared with (12). Note that the above functions $I\left(t_{1}, t_{2}, x\right)$ and $k\left(t_{1}, t_{2}\right)$ admit explicit expressions which can be used to more precisely compute the quantities $\left\langle b_{2}^{*}[\phi], b_{2}^{*}[\psi]\right\rangle_{L^{2}\left(S, \mathbb{R}^{2}\right)}$ in Lemma 4.

\subsubsection{Forward problems, $b_{2}\left[\mathbf{e}_{i}\right], b_{2}[\mathbf{m}]$}

Concerning the computation of the right-hand sides $\left\langle\mathbf{e}, b_{2}^{*}\left[g_{k}\right]\right\rangle_{L^{2}\left(S, \mathbb{R}^{2}\right)}$ of the critical point equations, following the definition of the operator $b_{2}$ in Section 3 ,

$$
b_{2}[\mathbf{m}]=-\left(P_{h}^{\prime} \star \widetilde{m_{1}}-Q_{h}^{\prime} \star \widetilde{m_{2}}\right)_{\left.\right|_{K}}, \quad \forall \mathbf{m}=\left(m_{1}, m_{2}\right) \in L^{2}\left(S, \mathbb{R}^{2}\right),
$$

we first compute, from the above definition of $\mathbf{e}_{i}$ for $i=1,2$ :

$$
\begin{gathered}
\left\langle\mathbf{e}_{1}, b_{2}^{*}\left[g_{k}\right]\right\rangle_{L^{2}\left(S, \mathbb{R}^{2}\right)}=\left\langle b_{2}\left[\mathbf{e}_{1}\right], g_{k}\right\rangle_{L^{2}(K)} \\
=\int_{-s}^{s} \int_{-q}^{q} P_{h}^{\prime}(x-t) e^{i k \pi \frac{t}{q}} d t d x=\int_{-q}^{q}\left(P_{h}(s-t)-P_{h}(s+t)\right) e^{i k \pi \frac{t}{q}} d t
\end{gathered}
$$

and

$$
\begin{gathered}
\left\langle\mathbf{e}_{2}, b_{2}^{*}\left[g_{k}\right]\right\rangle_{L^{2}\left(S, \mathbb{R}^{2}\right)}=\left\langle b_{2}\left[\mathbf{e}_{2}\right], g_{k}\right\rangle_{L^{2}(K)} \\
=\int_{-s}^{s} \int_{-q}^{q} Q_{h}^{\prime}(x-t) e^{i k \pi \frac{t}{q}} d t d x=\int_{-q}^{q}\left(Q_{h}(s-t)-Q_{h}(s+t)\right) e^{i k \pi \frac{t}{q}} d t .
\end{gathered}
$$

These expressions are computed by discretization on a family of piecewise constant functions on $K=\cup_{l} K_{l}$, summing up the contributions on $K_{l}$, although they are linked to the Fourier coefficients of $\chi_{K} P_{h}$ and $\chi_{K} Q_{h}$.

This is all we need in order to solve the linear systems (12) in $c_{n}$ and (14) in $d_{n}$, and to compute the solutions $\phi_{i}$ related to $\mathbf{e}_{i}$ and $M$.

In order to generate synthetic data, the components $m_{i}$ of magnetizations $\mathbf{m} \in L^{2}\left(S, \mathbb{R}^{2}\right)$ are also modelled as finite linear combinations of a family of piecewise constant functions on $S=\cup_{l} S_{l}, l=1, \cdots L$.

The quantity $b_{2}[\mathbf{m}]$ is computed from its Fourier series, while its Fourier coefficients (equal to $\left.\left\langle b_{2}[\mathbf{m}], g_{k}\right\rangle_{L^{2}(S)}\right)$ are computed as those of $b_{2}\left[\mathbf{e}_{i}\right]$ above, summing up the contributions of the intervals $S_{l}, l=1, \cdots L$.

We finally compute (with a quadrature method):

$$
\left\langle b_{2}[\mathbf{m}], \phi_{i}\right\rangle_{L^{2}(K)}=\sum_{n \in \mathbb{Z}} c_{n}\left\langle b_{2}[\mathbf{m}], g_{n}\right\rangle_{L^{2}(K)}
$$

which is expected to furnish the approximation $\left\langle m_{i}^{e}\right\rangle=\left\langle b_{2}[\mathbf{m}], \phi_{i}\right\rangle_{L^{2}(K)}$ to $\left\langle m_{i}\right\rangle$. For error estimation, we will compare $\left\langle m_{i}^{e}\right\rangle$ to the actual moments $\left\langle m_{i}\right\rangle(i=1,2)$. 


\subsection{Numerical illustrations}

In this section, we discuss preliminary numerical results concerning the computation of $\left\langle m_{i}^{e}\right\rangle=\left\langle b_{2}[\mathbf{m}], \phi_{i}\right\rangle_{L^{2}(K)}$ in order to estimate the mean value $\left\langle m_{i}\right\rangle$ of each component of $\mathbf{m}$. Recall that the solutions to $\left(\mathrm{CPE}_{m o}\right)$ in $L^{2}(K), \phi_{i}=\phi_{o}\left[\mathbf{e}_{i}\right], i=1,2$, are given by:

$$
\left[-P_{h}^{\prime} \star\left(\chi_{S}\left(P_{h}^{\prime} \star \widetilde{\phi}_{i}\right)\right)+Q_{h}^{\prime} \star\left(\chi_{S}\left(Q_{h}^{\prime} \star \widetilde{\phi}_{i}\right)\right)\right]_{\left.\right|_{K}}+\lambda \phi_{i}=\left\{\begin{array}{l}
-\left(P_{h}^{\prime} \star \chi_{S}\right)_{\left.\right|_{K}} i=1 \\
\left(P_{h}^{\prime} \star \mathcal{H} \chi_{S}\right)_{\left.\right|_{K}} i=2
\end{array}\right.
$$

for $\lambda>0$; we have $\left\|\phi_{i}\right\|_{L^{2}(K)}=M(\lambda)$.

The solutions $\phi_{i}=\phi_{o}\left[\mathbf{e}_{i}\right], i=1,2$, to $\left(\mathrm{CPE}_{m o, W}\right)$ in $W_{0}^{1,2}(K)$ are given by:

$$
\left[-P_{h}^{\prime} \star\left(\chi_{S}\left(P_{h} \star \widetilde{\phi_{i}^{\prime}}\right)\right)+Q_{h}^{\prime} \star\left(\chi_{S}\left(Q_{h} \star{\widetilde{\phi_{i}^{\prime}}}_{i}\right)\right]_{\left.\right|_{K}}-\lambda \phi_{i}^{\prime \prime}=\left\{\begin{array}{c}
-\left(P_{h}^{\prime} \star \chi_{S}\right)_{\left.\right|_{K}} i=1, \\
\left(P_{h}^{\prime} \star \mathcal{H} \chi_{S}\right)_{\left.\right|_{K}} i=2,
\end{array}\right.\right.
$$

with $\lambda=-\gamma>0$, and yet $\left\|\phi_{i}\right\|_{W_{0}^{1,2}(K)}=M(\lambda)$, in the weak sense in $L^{2}(K)$.

The quantities (coefficients) $\left\langle b_{2}^{*}\left[g_{n}\right], b_{2}^{*}\left[g_{k}\right]\right\rangle_{L^{2}\left(S, \mathbb{R}^{2}\right)}$ in (12) and (14) are computed with $f f t$ (fast Fourier transform), following Lemma 4, while for numerical purposes, the series will be truncated at some order $N \in \mathbb{N}_{*}$.

Various situations can be examined, with respect to the distance $h$ between the magnetization support $\bar{S}$ contained in $\mathbb{R} \times\{0\}$ and the measurement set $K \subset \mathbb{R} \times\{h\}$, and to their length $|S|,|K|$. In order to match with the physics of the model, we assume that $S=(-s, s)$ and $K=(-q, q)$ are centered at the origin, and that $|K|>|S|>h>0$, with (approximately) $|K| \simeq 1.5|S|,|S| \simeq 20 h$. We will take $h=0.1, s=1$ and $q=1.5$. The order of truncation will be $N=250$.

We will compare the estimates $\left\langle m_{i}^{e}\right\rangle$ of $\left\langle m_{i}\right\rangle$ for functions $\phi_{i}$ solutions to the BEP in $L^{2}$ or in $W_{0}^{1,2}$, associated to particular values of $\lambda$, for some magnetizations $\mathbf{m}$. The computations are performed with Matlab, R2017a.

Tables below furnish the estimated moments $\left\langle m_{i}^{e}\right\rangle$ for $i \in\{1,2\}$, the corresponding values of the relative errors $\varepsilon_{i}$ and norm constraints $M,\left\|\phi_{i}\right\|_{L^{2}(K)}$ or $\left\|\phi_{i}\right\|_{W_{0}^{1,2}(K)}$. Figures are provided in the Appendix.

Below, for the computation of the solutions $\phi_{i}$ to the corresponding BEP, we choose $\lambda=\lambda_{i}$ such that $1 \leq-\log _{10}\left(\lambda_{i}\right) \leq 9$ is an integer and such that the relative error for moments estimation given by

$$
\varepsilon_{i}=\frac{\left|\left\langle m_{i}\right\rangle-\left\langle m_{i}^{e}\right\rangle\right|}{\left|\left\langle m_{i}\right\rangle\right|}
$$

is small enough among this range for the corresponding data $\mathbf{m}$, while still providing an acceptable value for the quantity $M\left(\lambda_{i}\right)$, say between 10 and 20 , see Table 1 . Observe that this trade off is made possible by the fact that the data are available for the present simulations. Elsewhere, in general, $\lambda=\lambda_{i}$ has to be chosen in terms of the behaviour of the error $\left\|b_{2}^{*}\left[\phi_{i}\right]-\mathbf{e}_{i}\right\|_{L^{2}\left(S, \mathbb{R}^{2}\right)}$ (and of the associated constraint $M\left(\lambda_{i}\right)$ ), independently of the unknown magnetization $\mathbf{m}$.

In all the numerical experiments, the net moment will be kept fixed to: $\left\langle m_{1}\right\rangle=-0.1$ and $\left\langle m_{2}\right\rangle=0.1$, whence:

$$
\varepsilon_{i}=10\left|\left\langle m_{i}\right\rangle-\left\langle m_{i}^{e}\right\rangle\right|=\left|(-1)^{i-1}+10\left\langle m_{i}^{e}\right\rangle\right| .
$$

\subsubsection{Solutions $\phi_{i}$ to BEP}

The solutions $\phi_{i} \in L^{2}(K)$ for $i=1,2$ are plotted in Figure 5, $6\left(\lambda_{i}=10^{-3}\right.$ and $\left.10^{-5}\right)$. The solutions $\phi_{i} \in W_{0}^{1,2}(K)$ can be seen in Figures $7,8\left(\lambda_{i}=10^{-8}\right.$ and $\left.10^{-9}\right)$. Their norms are given in Table 1. 


\begin{tabular}{|c|c|c|c|}
\hline$\phi_{i}$ & $\lambda_{i}$ & $\left\|\phi_{1}\right\|$ & $\left\|\phi_{2}\right\|$ \\
\hline in $L^{2}(K)$ & $10^{-3}$ & 4.8 & 4.4 \\
\hline & $10^{-5}$ & 14.4 & 8.2 \\
\hline in $W_{0}^{1,2}(K)$ & $10^{-8}$ & 19.9 & 10.4 \\
\hline & $10^{-9}$ & 645.5 & 221.7 \\
\hline
\end{tabular}

Table 1: Values of $\lambda_{i}, M\left(\lambda_{i}\right)=\left\|\phi_{i}\right\|_{L^{2}(K)}$ or $\left\|\phi_{i}\right\|_{W_{0}^{1,2}(K)}, i=1,2$.

The solutions in $L^{2}(K)$ show quite many oscillations close to the boundary of $S$, less in $W_{0}^{1,2}(K)$, as expected. Interestingly, these functions behave as affine or constant in a large interval contained in the interior of $S$. We also see that as $\lambda_{i}$ decreases, the corresponding constraint $M\left(\lambda_{i}\right)$ grows, which also increases the oscillating phenomenon, mainly in $L^{2}(K)$.

\subsubsection{Constant magnetizations}

Take $m_{1}=-0.05$ on $S=[-1,1]$ and $m_{2}=0.05$ on $S$ (whence $\mathbf{m}=0.05\left(\mathbf{e}_{1}-\mathbf{e}_{2}\right)$ and $\left.m_{i}=0.05(-1)^{i} \chi_{S}\right)$. See Figure 1 and Table 2 for plots of $m_{i}$ and of $b_{2}[\mathbf{m}]$, computed as explained in Section 5.1.3.

\begin{tabular}{|c|c|c|c|c|c|}
\hline$\phi_{i}$ & $\lambda_{i}$ & $\left\langle m_{1}^{e}\right\rangle$ & $\left\langle m_{2}^{e}\right\rangle$ & $\varepsilon_{1}$ & $\varepsilon_{2}$ \\
\hline in $L^{2}(K)$ & $10^{-5}$ & -0.1044 & 0.09581 & $4.4 \cdot 10^{-4}$ & $4.2 \cdot 10^{-3}$ \\
\hline in $W_{0}^{1,2}(K)$ & $10^{-8}$ & -0.0996 & 0.0994 & $3.8 \cdot 10^{-3}$ & $6.4 \cdot 10^{-3}$ \\
\hline
\end{tabular}

Table 2: $\mathbf{m}$ identically constant.

\subsubsection{Magnetizations with large support}

Take $m_{1}=-0.1$ on $[-1,0], 0$ elsewhere in $S$, and $m_{2}=0.1$ on $[0,1]$, see Figure 2 , together with Table 3.

\begin{tabular}{|c|c|c|c|c|c|}
\hline$\phi_{i}$ & $\lambda_{i}$ & $\left\langle m_{1}^{e}\right\rangle$ & $\left\langle m_{2}^{e}\right\rangle$ & $\varepsilon_{1}$ & $\varepsilon_{2}$ \\
\hline in $L^{2}(K)$ & $10^{-5}$ & -0.0999 & 0.0994 & $6.4 \cdot 10^{-4}$ & $5.5 \cdot 10^{-3}$ \\
\hline in $W_{0}^{1,2}(K)$ & $10^{-8}$ & -0.1000 & 0.0995 & $4.4 \cdot 10^{-4}$ & $4.6 \cdot 10^{-3}$ \\
\hline
\end{tabular}

Table 3: $\mathbf{m}$ with large support.

\subsubsection{Other magnetization}

Here we take $m_{1}$ and $m_{2}$ as below. See Figure 3 and Table 4 .

$$
m_{1}=\left\{\begin{array}{ll}
-0.05 & \text { on }[-0.2,0], \\
-0.1 & \text { on }[0,0.2], \\
-0.2 & \text { on }[0.2,0.4], \\
-0.1 & \text { on }[0.4,0.6], \\
-0.05 & \text { on }[0.6,0.8], \\
0 & \text { elsewhere in } S,
\end{array} \quad m_{2}= \begin{cases}0.05 & \text { on }[-0.8,-0.6], \\
0.1 & \text { on }[-0.6,-0.4], \\
0.2 & \text { on }[-0.4,-0.2], \\
0.1 & \text { on }[-0.2,0] \\
0.05 & \text { on }[0,0.2], \\
0 & \text { elsewhere in } S .\end{cases}\right.
$$




\begin{tabular}{|c|c|c|c|c|c|}
\hline$\phi_{i}$ & $\lambda_{i}$ & $\left\langle m_{1}^{e}\right\rangle$ & $\left\langle m_{2}^{e}\right\rangle$ & $\varepsilon_{1}$ & $\varepsilon_{2}$ \\
\hline in $L^{2}(K)$ & $10^{-5}$ & -0.0981 & 0.09855 & $1.9 \cdot 10^{-2}$ & $1.4 \cdot 10^{-2}$ \\
\hline in $W_{0}^{1,2}(K)$ & $10^{-8}$ & -0.0977 & 0.0989 & $2.3 \cdot 10^{-2}$ & $1.1 \cdot 10^{-2}$ \\
\hline
\end{tabular}

Table 4: Other $\mathbf{m}$.

\subsubsection{Magnetizations with small support}

Here we take $m_{1}$ and $m_{2}$ as described below. See Figure 4 and Table 5 .

$$
m_{1}=\left\{\begin{array}{ll}
10 & \text { on }[-0.5,-0.49], \\
-10 & \text { on }[0,0.0 .1], \\
-10 & \text { on }[0.2,0.21], \\
0 & \text { elsewhere in } S,
\end{array} \quad m_{2}= \begin{cases}10 & \text { on }[-0.9,-0.89], \\
-10 & \text { on }[-0.3,-0.29] \\
10 & \text { on }[0.2,0.21], \\
0 & \text { elsewhere in } S .\end{cases}\right.
$$

\begin{tabular}{|c|c|c|c|c|c|}
\hline$\phi_{i}$ & $\lambda_{i}$ & $\left\langle m_{1}^{e}\right\rangle$ & $\left\langle m_{2}^{e}\right\rangle$ & $\varepsilon_{1}$ & $\varepsilon_{2}$ \\
\hline in $L^{2}(K)$ & $10^{-5}$ & -0.104 & 0.0958 & $4.4 \cdot 10^{-2}$ & $4.2 \cdot 10^{-2}$ \\
\hline in $W_{0}^{1,2}(K)$ & $10^{-8}$ & -0.1015 & 0.0969 & $1.5 \cdot 10^{-2}$ & $3.1 \cdot 10^{-2}$ \\
\hline
\end{tabular}

Table 5: m with small support.

\subsubsection{Comments, discussion}

Overall, as presented in the above tables, we obtain quite accurate results for net moment estimation. The net moments of magnetizations with large support are more precisely estimated than those of magnetizations with small support: the value $\varepsilon_{i}$ of the error decreases whenever the size of the support increases, for a same value of the parameter $\lambda_{i}$. This phenomenon is mostly true in $L^{2}(K)$ for the present examples. These examples may not always provide smaller estimation errors $\varepsilon_{i}$ in $W_{0}^{1,2}(K)$ than in $L^{2}(K)$, since the behaviour of $\varepsilon_{i}$ depend on the specific data (smoothness properties of $\mathbf{m}$, size of its support, ...) and parameters. These properties, together with the influence of the noise in the computations and in the data, will be studied in a forthcoming work.

\section{Perspectives, conclusions}

In order to complete the results of Section 3, some properties of $b_{2}$ remain to be studied. Even if $b_{2}$ is injective, it is not coercive (strongly injective), in the sense that $\left\|b_{2}[\mathbf{m}]\right\|_{L^{2}(K)}$ can be small even when $\|\mathbf{m}\|_{L^{2}\left(S, \mathbb{R}^{2}\right)}$ is not small: there may exist such "almost silent" source terms $\mathbf{m}$. However, lower bounds for $\left\|b_{2}\right\|$ can be established if we restrict to truncated Fourier expansions, showing that these cannot be "almost silent". Also, one can estimate the constants involved in the upper bounds of $\left\|b_{2}\right\|$ and $\left\|b_{2}^{*}\right\|$. The spectral study of the operator $b_{2} b_{2}^{*}$ remains to be fulfilled, in view of the numerical analysis of the BEP.

The BEP studied in Section 4 can be stated with slightly more general constraints. In particular, let $f \in L^{2}(K)$. Consider the following BEP in $L^{2}(K)$ :

$$
\min _{\substack{\phi \in L^{2}(K) \\\|\phi-f\|_{L^{2}(K)} \leq M}}\left\|b_{2}^{*}[\phi]-\mathbf{e}\right\|_{L^{2}\left(S, \mathbb{R}^{2}\right)} .
$$

One can prove existence and uniqueness of the function $\phi_{o} \in L^{2}(K)$ solution of $\left(\mathrm{BEP}_{f}\right)$ using the same arguments as in the proof of Proposition 6. Moreover, if $\mathbf{e} \notin \operatorname{Ran} b_{2}^{*}$ (or 
if $\mathbf{e}=b_{2}^{*}[\phi]$ for some $\phi \in L^{2}(K)$ such that $\left.\|\phi-f\|_{L^{2}(K)} \geq M\right)$, then the solution $\phi_{o}$ still saturates the constraint, i.e. $\left\|\phi_{o}-f\right\|_{L^{2}(K)}=M$, and is given by the following implicit equation, with $\gamma<0$, see $\left(\mathrm{BEP}_{m o}\right)$ :

$$
b_{2} b_{2}^{*}\left[\phi_{o}\right]-\gamma \phi_{o}=b_{2}[\mathbf{e}]+\gamma f .
$$

Let now $f \in W_{0}^{1,2}(K)$. The BEP can be stated as follows:

$$
\min _{\substack{\phi \in W_{0}^{1,2}(K) \\\|\phi-f\|_{W_{0}^{1,2}(K)}^{1,2} \leq M}}\left\|b_{2}^{*}[\phi]-\mathbf{e}\right\|_{L^{2}\left(S, \mathbb{R}^{2}\right)} \cdot \quad\left(\mathrm{BEP}_{f, W}\right)
$$

Again, as for $\left(\mathrm{BEP}_{m o, W}\right)$, a solution $\phi_{o} \in W_{0}^{1,2}(K)$ exists, is unique, and saturates the constraint if $\mathbf{e} \notin b_{\left.2\right|_{W}}^{*}\left[W_{0}^{1,2}(K)\right]$ (or if $\mathbf{e}=b_{\left.2\right|_{W}}^{*}[\phi]$ for $\phi \in L^{2}(K)$ such that $\|\phi-f\|_{W_{0}^{1,2}(K)} \geq$ $M):\left\|\phi_{o}-f\right\|_{W_{0}^{1,2}(K)}=M$. It satisfies the following equation, with $\gamma<0$ :

$$
b_{2} b_{2}^{*}\left[\phi_{o}\right]+\gamma \phi_{o}^{\prime \prime}=b_{2}[\mathbf{e}]+\gamma f .
$$

The above problem will be considered for $\phi_{o} \in W^{1,2}(K)$ with norm constraint therein, where it is also well-posed and gives promising numerical results. Moreover, $W^{1,2}(K)$ functions are continuous on $K$ in the present 1-dimensional setting, which is a suitable property of the solution, in view of computing its inner product with the available measurements in a pointwise way. Note however that the continuity of the solution is already granted by the critical point equation $\left(\mathrm{CPE}_{m o}\right)$ above and the smoothness properties of functions in $\operatorname{Ran} b_{2}$. Some BEP of mixed type, with constraints in other norms, like uniform, $L^{1}$, BMO, could also be formulated, as well as related extremal problems consist in looking for the best bounded extension of a given data $b_{d} \simeq b_{2}[\mathbf{m}]$ from $K$ to $\mathbb{R}$.

Following Section 5, further numerical analysis and improvements of the computational schemes will be considered. In particular, we expect that refined implementations of the computations in the Fourier domain will provide more efficient and accurate recovery schemes, in the present setting as well as in the $3 \mathrm{D}$ one, see [6]. Both a quantitative and a qualitative study of the relations $\lambda \mapsto M(\lambda)$ and $\lambda \mapsto \epsilon_{i}(\lambda)$ (for the different error terms) remain to be done. This will be the topic of a further work, together with a study concerning the influence of the parameters $s, q, h$, and of the characteristics of $m_{i}$, on the behaviour of the solutions.

Both explicit and asymptotic expressions, in terms of the size $q$ of the measurement set $K$, relating the first moments of $\mathbf{m}$ to those of $b_{2}[\mathbf{m}]$, could also be derived, as in [8] for the 3D situation. Besides, the quantities $b_{2}^{*}\left[x^{k}\right]$ for monomials $x^{k}$ could be exactly computed.

Other functions e may be considered as well, both for the present net moment estimation problem or for higher order or local moments recovery. One can also take the components $e_{i}$ to be appropriate band-limited basis functions, of Slepian type [22].

Particularly interesting magnetizations are the unidirectional ones, of which the moment recovery using the above linear estimator process still requires a specific study. Also, more general situations where $\mathbf{m}$ belongs to $L^{1}\left(S, \mathbb{R}^{2}\right)$ or is a measure remain to be considered, see [5], together with magnetizations supported on a two-dimensional set.

Finally, the full inversion problem of recovering $\mathbf{m}$ in $L^{2}\left(S, \mathbb{R}^{2}\right)$ itself from $b_{2}[\mathbf{m}]$ will be considered in a further work. It can also be stated as a BEP of which the solution will involve the operator $b_{2} b_{2}^{*}$. Again more general magnetization distributions will be studied.

\section{Acknowledgments}

The authors warmly thank Jean-Paul Marmorat for his help in numerical implementations. 


\section{References}

[1] R. Alaifari Analysis of the truncated Hilbert transform arising in limited data computerized tomography, $\mathrm{PhD}$ thesis, University of Brussels, 2014.

[2] R. Alaifari, M. Defrise, A. Katsevich, Spectral analysis of the truncated Hilbert transform with overlap, SIAM J. Math. Anal., 46, 192-213, 2014.

[3] R. Alaifari, L.B. Pierce, S. Steinerberger, Lower bounds for the truncated Hilbert transform., Rev. Mat. Iberoamericana, 32, 23-56, 2016.

[4] S. Axler, P. Bourdon, W. Ramey, Harmonic Function Theory, Springer-Verlag, 2nd edition, 2001.

[5] L. Baratchart, D. P. Hardin, E. A. Lima, E. B. Saff, B. P. Weiss, Characterizing kernels of operators related to thin-plate magnetizations via generalizations of Hodge decompositions, Inverse Problems, 29 (1), 2013.

[6] L. Baratchart, S. Chevillard, D. Hardin, J. Leblond, E. A. Lima, J.-P. Marmorat, Magnetic moment estimation and bounded extremal problems, Inverse Problems \& Imaging, to appear, 13 (1), 2019 (hal.archives-ouvertes.fr/hal-01623991).

[7] L. Baratchart, S. Chevillard, J. Leblond, Silent and equivalent magnetic distributions on thin plates, Theta Series in Advanced Mathematics, Proceedings of the Conference "Analyse Harmonique et Fonctionnelle, Théorie des Opérateurs et Applications" 2015 (in honor of Jean Esterle), eds. Ph. Jaming, A. Hartmann, K. Kellay, S. Kupin, G. Pisier, D. Timotin, Theta Series in Advanced Mathematics, 11-28, 2017.

[8] L. Baratchart, S. Chevillard J. Leblond, E. A. Lima and D. Ponomarev. Asymptotic method for estimating magnetic moments from field measurements on a planar grid. In preparation.

[9] H. Brezis, Functional Analysis, Sobolev Spaces and Partial Differential Equations, Springer, 2011.

[10] I. Chalendar, J. Leblond, J. R. Partington, Approximation problems in some holomorphic spaces, with applications, Systems, Approximation, Singular Integral Operators, and Related Topics, Proceedings of IWOTA 2000, eds. A.A. Borichev, N.K. Nikolski, Integral Equations and Operator Theory 129, 143-169, 2001.

[11] I. Chalendar, J. R. Partington, Constrained approximation and invariant subspaces, J. Math. Anal. Appl., 280, 176-187, 2003.

[12] I. Chalendar J. R. Partington, Modern approaches to the invariant-subspace problem, Cambridge Tracts in Mathematics, 188, Cambridge University Press, 2011.

[13] P. L. Duren, Theory of $H^{p}$ spaces, Pure and Applied Mathematics, 38, 1970.

[14] J.B. Garnett, Bounded analytic functions, Graduate Texts in Mathematics, 236, Springer, 2007.

[15] J. D. Jackson, Classical Electrodynamics, 3rd ed., Wiley, 1999.

[16] B. Jacob, J. Leblond, J.-P. Marmorat, J. R. Partington, A constrained approximation problem arising in parameter identification, Linear Algebra and its Applications, 351-352, 487-500, 2002.

[17] P. Jaming, E. Pozzi, B. D. Wick, Lower bounds for the dyadic Hilbert transform, Annales de la Faculté des Sciences de Toulouse, 27, 265-284, 2018.

[18] T. Kato, Perturbation Theory for Linear Operators, Springer-Verlag, 132, 2nd ed., 1976.

[19] W. Koppelman and J. D. Pincus, Spectral Representations for Finite Hilbert Transformations, Mathematische Zeitschrift, 71, 399-407, 1959.

[20] R. Kress, Linear Integral Equations, Springer-Verlag, 1989. 
[21] J. Leblond, D. Ponomarev, On some extremal problems for analytic functions with constraints on real or imaginary parts, Festschrift in Honor of Daniel Alpay's 60th Birthday, F. Colombo \& al. (eds.), Advances in Complex Analysis and Operator Theory, Trends in Math., Birkhäuser, 219-236, 2017.

[22] J. Ledford, Recovery of bivariate band-limited functions using scattered translates of the Poisson kernel, Journal of Approximation Theory, 189, 170-180, 2015.

[23] J. Mashreghi, Representation Theorems in Hardy Spaces, London Mathematical Society Student Texts, Cambridge University Press, Cambridge, 74, 2009.

[24] N. K. Nikolski, Operators, functions, and systems: an easy reading. Vol. 1, Mathematical Surveys and Monographs, 92, American Mathematical Society, Providence, RI, 2002.

[25] D. Ponomarev, Some inverse problems with partial data, PhD thesis, Université Nice Sophia Antipolis, 2016.

[26] W. Rudin, Functional Analysis, McGraw-Hill, 1991.

[27] E. M. Stein, G. Weiss, Introduction to Fourier analysis on Euclidean spaces, Princeton Univ. Press, 1971. 


\section{Appendix: numerical illustrations}
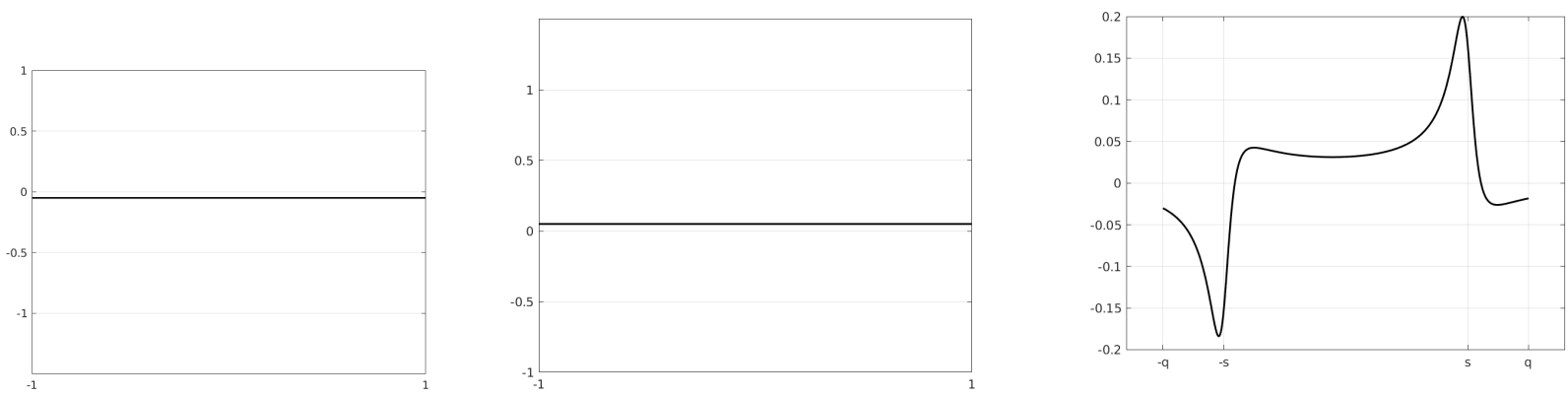

Figure 1: $m_{1}, m_{2}$ constant, associated $b_{2}[\mathbf{m}]$.
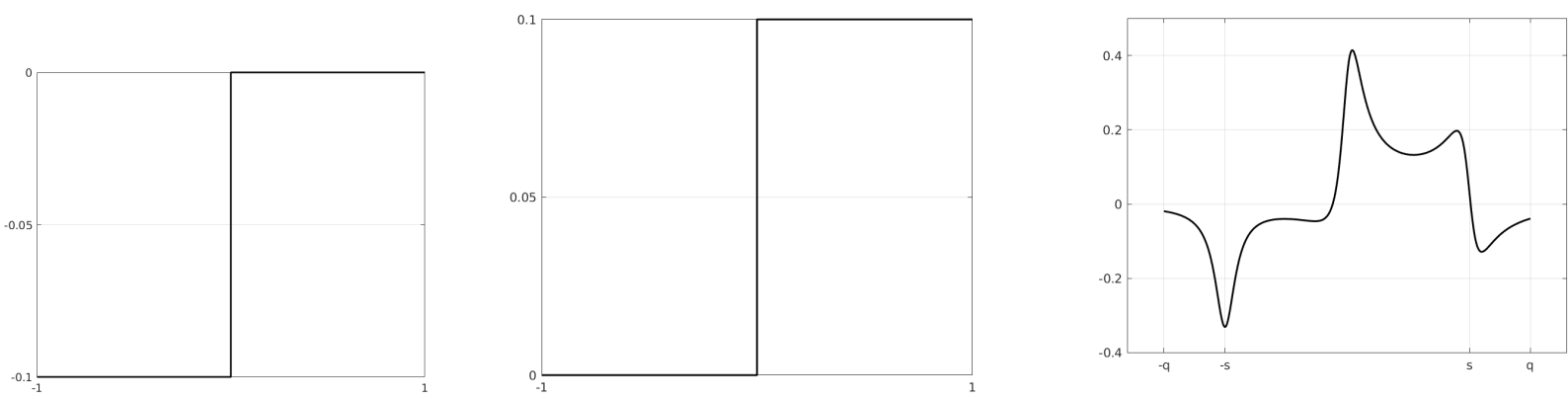

Figure 2: $m_{1}, m_{2}$ with large support, associated $b_{2}[\mathbf{m}]$.
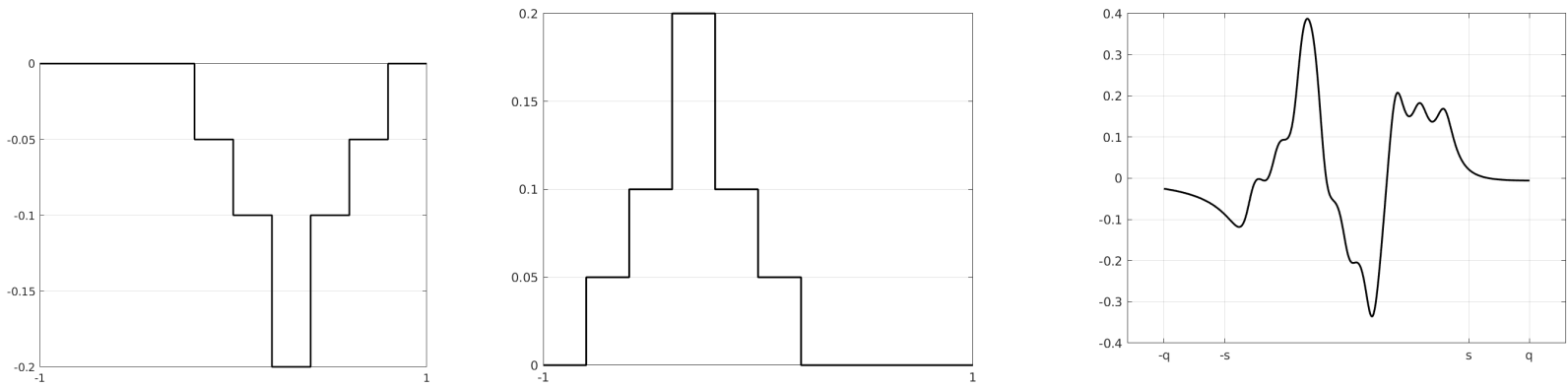

Figure 3: Others $m_{1}, m_{2}$, associated $b_{2}[\mathbf{m}]$. 

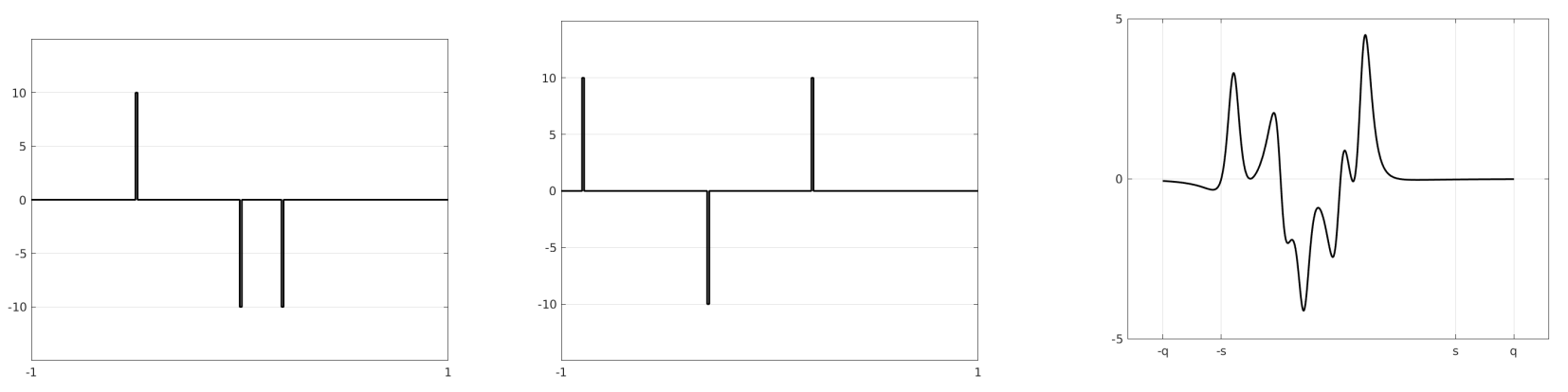

Figure 4: $m_{1}, m_{2}$ with small support, associated $b_{2}[\mathbf{m}]$.
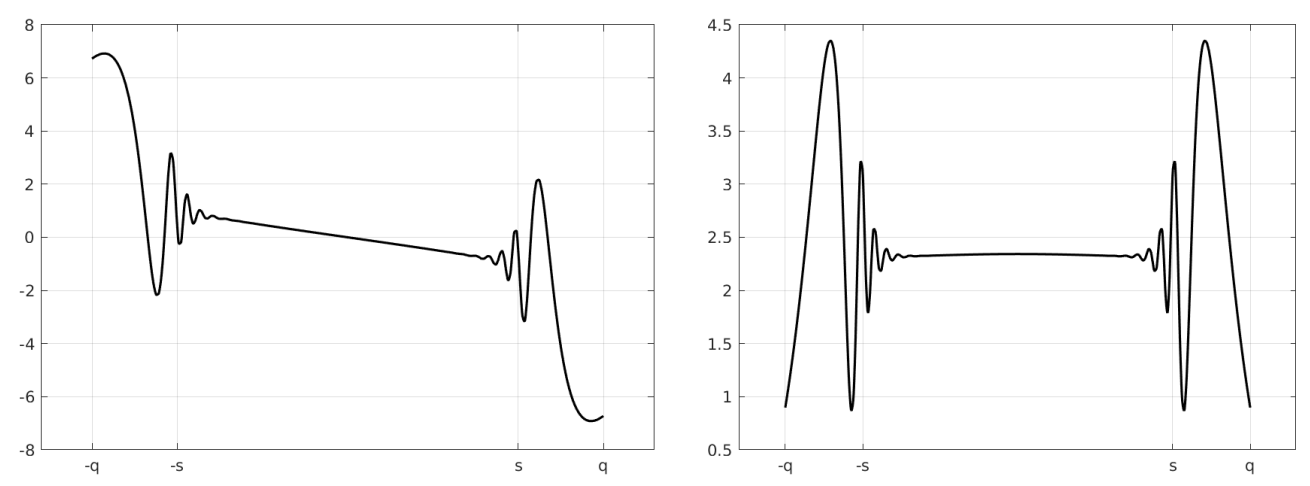

Figure 5: Solutions $\phi_{i} \in L^{2}(K)$ for $i=1,2, \lambda_{i}=10^{-3}$.
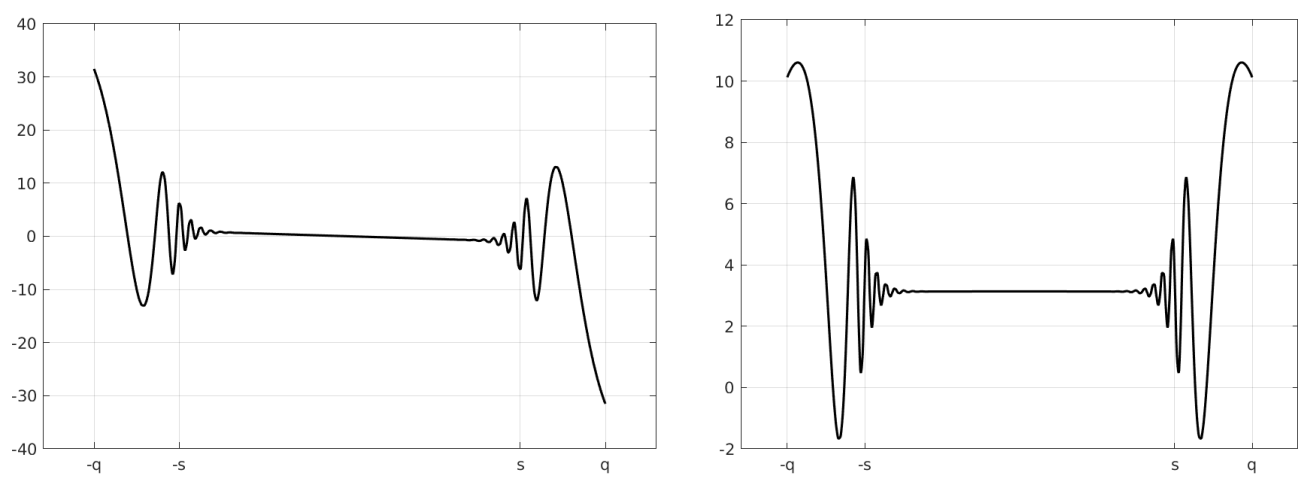

Figure 6: Solutions $\phi_{i} \in L^{2}(K)$ for $i=1,2, \lambda_{i}=10^{-5}$. 

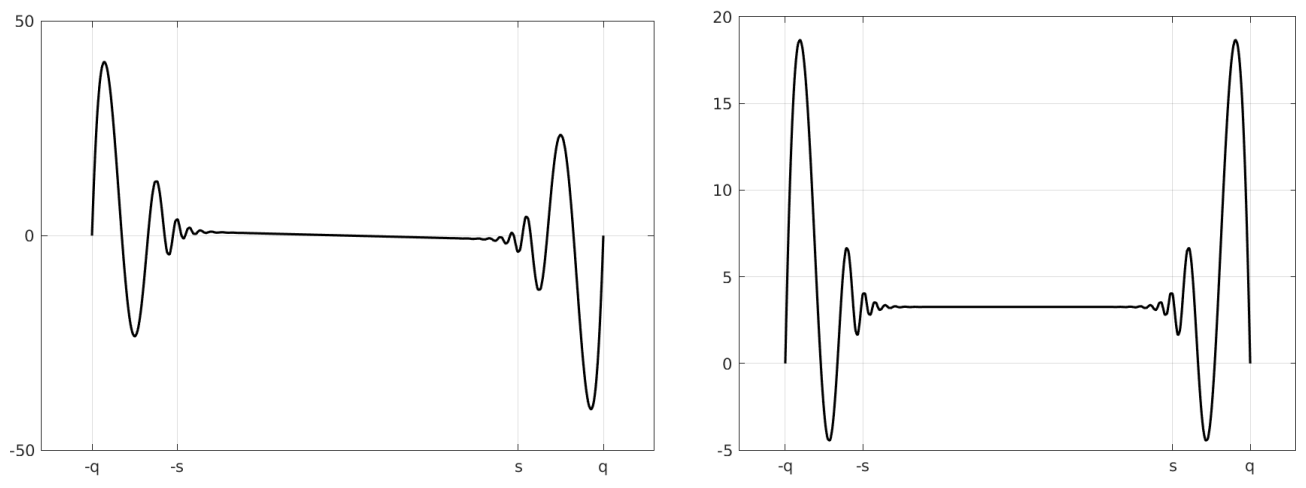

Figure 7: Solutions $\phi_{i} \in W_{0}^{1,2}(K), i=1,2, \lambda_{i}=10^{-8}$.
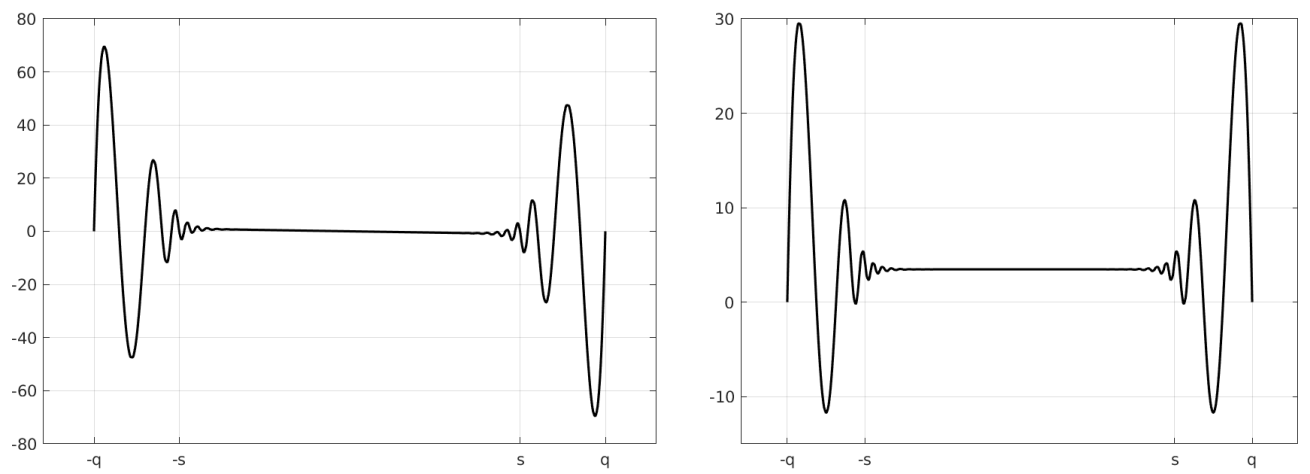

Figure 8: Solutions $\phi_{i} \in W_{0}^{1,2}(K), i=1,2, \lambda_{i}=10^{-9}$. 